\title{
Signalling involving MET and FAK supports cell division independent of the activity of the cell cycle-regulating CDK4/6 kinases
}

\author{
Chi Zhang ${ }^{1,2} \cdot$ Simon R. Stockwell $\mathbb{D}^{1} \cdot$ May Elbanna $^{1} \cdot$ Robin Ketteler $^{3} \cdot$ Jamie Freeman $^{3} \cdot$ Bissan Al-Lazikani $^{2}$. \\ Suzanne Eccles ${ }^{2} \cdot$ Alexis De Haven Brandon ${ }^{2}$ - Florence Raynaud ${ }^{2} \cdot$ Angela Hayes $^{2} \cdot$ Paul A. Clarke $\mathbb{C}^{2}$. \\ Paul Workman $\mathbb{( i )}^{2} \cdot$ Sibylle Mittnacht $\left(\mathbb{D}^{1}\right.$
}

Received: 16 February 2019 / Revised: 7 May 2019 / Accepted: 13 May 2019 / Published online: 12 July 2019

(c) The Author(s) 2019. This article is published with open access

\begin{abstract}
Deregulation of cyclin-dependent kinases 4 and $6(\mathrm{CDK} 4 / 6)$ is highly prevalent in cancer; yet, inhibitors against these kinases are currently used only in restricted tumour contexts. The extent to which cancers depend on CDK4/6 and the mechanisms that may undermine such dependency are poorly understood. Here, we report that signalling engaging the MET proto-oncogene receptor tyrosine kinase/focal adhesion kinase (FAK) axis leads to CDK4/6-independent CDK2 activation, involving as critical mechanistic events loss of the CDKI p21 ${ }^{\mathrm{CIP} 1}$ and gain of its regulator, the ubiquitin ligase subunit SKP2. Combined inhibition of MET/FAK and CDK4/6 eliminates the proliferation capacity of cancer cells in culture, and enhances tumour growth inhibition in vivo. Activation of the MET/FAK axis is known to arise through cancer extrinsic and intrinsic cues. Our work predicts that such cues support cell division independent of the activity of the cell cycle-regulating CDK4/6 kinases and identifies MET/FAK as a tractable route to broaden the utility of CDK4/6 inhibitor-based therapies in the clinic.
\end{abstract}

\section{Introduction}

The cyclin-dependent kinases CDK4 and CDK6 are core components of the signal transduction network controlling transition of cells from G1 (Gap1) phase of the cell cycle into $\mathrm{S}$ (DNA synthesis) [1,2]. Deregulation of this network is a common event in cancer. Multiple oncogenic pathways promote the synthesis of the activator D-type cyclins, and

Supplementary information The online version of this article (https:// doi.org/10.1038/s41388-019-0850-2) contains supplementary material, which is available to authorized users.

$\triangle$ Paul Workman

paul.workman@icr.ac.uk

$\triangle$ Sibylle Mittnacht

s.mittnacht@cancer.ucl.ac.uk

1 UCL Cancer Institute, University College London, London WC1E 6DD, UK

2 Cancer Research UK Cancer Therapeutics Unit at The Institute of Cancer Research, London SM2 5NG, UK

3 MRC Laboratory for Molecular Cell Biology, University College London, London WC1E 6BT, UK gene mutation of regulators involved in limiting CDK4/6 activation is exceptionally frequent in all types of cancer $[3,4]$. The high frequency by which CDK4/6 regulation is compromised in cancer implies that CDK4/6 deregulation is a key event enabling cancer development, and by extension, that inhibition of CDK $4 / 6$ could be a broadly applicable and effective approach to cancer treatment $[2,5]$.

Several potent, selective small-molecule inhibitors targeting CDK4/6 (CDK4/6is) have undergone clinical trials, including palbociclib (PD0332991), abemaciclib (LY2835219) and ribociclib (LEE001) [6, 7], and gained regulatory approval in combination with hormonal therapy in breast cancer [8-11]. However, evidence for clinical benefit has not been extended to other cancer types thus far, and relapse under therapy is frequent in the approved indication in breast cancer [9].

Activation of CDK4/6 requires their binding to D-type cyclins, synthesised in response to mitogenic signals $[12,13]$. Bound to these cyclins, CDK $4 / 6$ phosphorylates the retinoblastoma tumour suppressor protein (RB1), initiating its inactivation. RB1 in its active form prevents the transcription of genes required for S-phase entry, including those encoding the E- and A-type cyclins involved in the activation of the CDK4/6-related S phase cyclin-dependent 
kinase CDK2 [14]. In addition, RB1 promotes the ubiquitindependent destruction of the SCF (SKP1-CUL1-F-box protein) E3-ubiquitin-ligase substrate-recognition subunit SKP2, stabilizing the CDK2 inhibitory proteins $\mathrm{p} 21^{\mathrm{CIP} 1}$ and $\mathrm{p} 27^{\mathrm{KIP} 1}$, the degradation of which is SKP2 dependent $[15,16]$. Together, these activities limit CDK2 activation, safeguarding licensed DNA synthesis and cell cycle transit.

Numerous reports describe situations where activation of CDK2 is enabled in the absence of CDK4/6 activity and show that CDK2 can drive cycle transit in the absence CDK4/6 activity [17-24]. Hence, activation of CDK2, independent of CDK4/6 activity, may limit the potency of CDK4/6is in cancers, and identification of signalling required for $\mathrm{CDK} 2$ activation may yield information that predicts CDK4/6 inhibitor response, or be exploited to extend their efficacy in cancer therapy.

Here, we report a mechanism-focussed screen aimed at identifying signalling that enables CDK4/6-independent CDK2 activation. We identify a prominent role of the MET proto-oncogene tyrosine kinase receptor family and their downstream effectors, the focal adhesion kinase (FAK) family. Our data validate MET/FAK signalling as a mechanism that enables CDK4/6-independent CDK2 activation and cell cycle transit, and we provide evidence for the utility of MET or FAK inhibition as a means to improve tumour response to CDK4/6 inhibition in vivo.

\section{Results}

\section{Screening identifies proteins required for CDK2 activation in CDK4/6-inhibited cells}

To assess CDK2 activation in cells exposed to CDK4/6is, we used a cell-based CDK2 reporter (GFP-PSLD) where a green-fluorescent protein (GFP) is fused to the CDK2regulated phosphorylated subcellular localisation domain (PSLD) of human DNA helicase B [25]. Phosphorylation of the PSLD by CDK2 exposes a nuclear export sequence, initiating nuclear exclusion of the GFP fusion protein and accumulation in the cytoplasm (Fig. 1a). We made use of human colorectal carcinoma HCT116 cells (HCT116) that stably express GFP-PSLD (HCT116-PSLD) [26]. Treatment of HCT116-PSLD with siRNA targeting CDK2 or the CDK4/6i palbociclib significantly increased the fraction of cells, with predominantly nuclear fluorescence (nuclear: cytoplasmic (nuc/cyto) fluorescence ratio $>1.5$ ), consistent with reliance of reporter localisation on CDK2, and CDK4/ 6 activation (Supplementary Fig. 1a-c). However, although palbociclib treatment increased the percentage of cells with the nuclear-localised CDK2 reporter, a considerable portion of cells with loss of CDK4/6 activity, detected by the absence of RB1 phosphorylated at the CDK4/6-selective phosphorylation site Ser780 (pRB1 ${ }^{\mathrm{S} 780}$ ) [27], continued to contain a reporter with predominant cytoplasmic localisation (Supplementary Fig. 1d, e). This indicates CDK4/6-independent CDK2 activation. Unexpectedly, we observed that ablation of the tumour suppressor TP53 reduced CDK2 control by palbociclib, even though the cells remained responsive to CDK4/6 inhibition, indicated by the reduction in cells containing $\mathrm{pRB} 1^{\mathrm{S} 780}$ (Supplementary Fig. 1f and 1g).

To identify signalling that permits CDK4/6-independent CDK2 activation, we transfected HCT116-PSLD with a library of small- interfering RNA (siRNA) pools targeting kinases and kinase-relevant components and assessed the ability of palbociclib to restrain CDK2 activation under these conditions (Fig. 1b). Since we observed relaxed CDK2 control following ablation of TP53 and because functional TP53 loss is frequent in cancer, we included an arm to the screen where we compromised TP53 expression using TP53-targeted siRNA.

To identify siRNA pools that selectively decreased CDK2 activity subject to palbociclib treatment, we computed the sensitivity index (SI), which quantifies the difference between the expected combined and measured effects of two treatments $[28,29]$, in this case the effects of specific siRNAs and the effect of CDK4/6 inhibition on CDK2 activity. Ranking the SI values calculated using Z-score statistics (Fig. 1c), we selected siRNA pools with $\mathrm{Z}$ scores $>2$ for further analysis, yielding 30 pools that selectively decreased CDK2 in combination with CDK4/6 inhibition in HCT116-PSLD cells, and 23 in HCT116PSLD with compromised TP53 expression.

Most siRNA pools identified in TP53-compromised HCT116-PSLD cells decreased CDK2 activity also in HCT116-PSLD. Conversely, less than half identified in HCT116-PSLD decreased CDK2 activity in TP53-modified cells (Fig. 1d). These results indicate differences in the regulation of CDK2 in TP53-normal and TP53-impaired backgrounds, but at the same time, highlight the opportunity to enhance the dependence of CDK2 activation on CDK4/6 in cells, regardless of TP53 status.

\section{MET/FAK signalling is required for CDK2 activation in CDK4/6-inhibited cells}

To mine for annotated pathways overrepresented amongst the siRNA targets identified, we used the MetaCore ${ }^{\mathrm{TM}}$ GeneGO tool (Supplementary Tables 1 and 2). This revealed as most significantly enriched a well-connected hub involving the MET proto-oncogene/hepatocyte growth factor receptor (MET) and the closely related macrophage growth factor receptor (MST1R/RON), along with fibroblast growth factor receptor 3 (FGFR3) and their common downstream signalling targets, the focal adhesion kinases (FAK) PTK2 and PTK2B (Fig. 2a). 
Fig. 1 Screen for proteins permitting CDK2 activation in cells with CDK4/6 inhibition. a Schematic depicting functioning of the CDK2 reporter GFP-PSLD. Modular reporter structure, relationship between subcellular distribution of GFP and cell cycle phase, and a representative image of individual HCT116-PSLD with low (GFP-PSLD nuc/cyto $>1.5$ ) or high (GFP-PSLD nuc/cyto < 1.5) CDK2 activity is shown. HDHB, human DNA helicase B; NES, nuclear export sequence. b Screen outline and procedure for hit identification. $\mathbf{c}$ Z-score ranking for siRNA pools in the screen. Results for unperturbed (siNT) and TP53-perturbed (siTP53) conditions are shown. Data points represent the mean of $n=3$ independent repeats; siRNA pools with $\mathrm{Z}$ score $>2$ marked in red. d Hierarchical clustering of hits based on mean $(n=3)$ sensitivity index values (SI). Colours denote the nature of interaction between the siRNA pool and palbociclib: red, synergistic; white, additive; blue, antagonistic. siRNA target genes on the right. (Related to Supplementary Fig. 1)

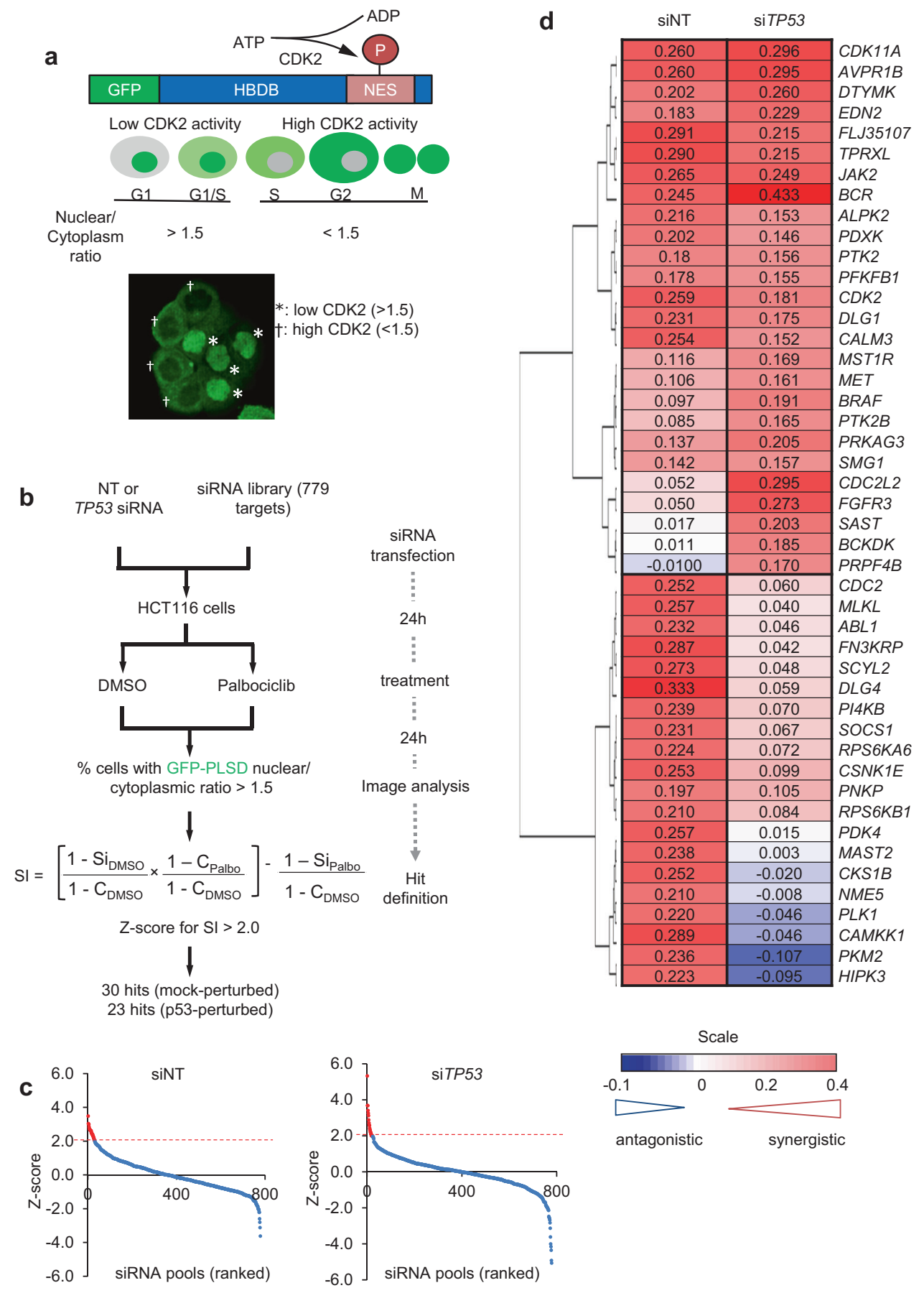

Two or more distinct siRNAs targeting MET, MST1R, PTK2 or PTK2B synergistically increased the percentage cells with nuclear-localised PSLD-GFP following CDK4/6 inhibition (Fig. 2b), validating the involvement of MET and FAK family members in enabling CDK4/6-independent CDK2 activation in cells. Combined use of $M E T$ and $M S T 1 R$ or PTK2 and PTK2B siRNA did not enhance the outcome, suggesting an independent, rate-limiting contribution of individual MET and FAK family kinases in this context. Notably, treatment with chemical inhibitors targeting either the MET or FAK family kinases synergistically decreased CDK2 activity in combination with palbociclib (Fig. 2c). The activity of network components FGFR3, SRC and JAK did not confirm with multiple oligonucleotides (Fig. 2b). Hence, the involvement of these components in enabling CDK4/6-independent CDK2 activation cannot be certain.

To assess if inhibition of MET enables CDK2 control by enhancing the efficacy of CDK4/6is to control CDK4/6, we assessed loss of $\mathrm{pRB}^{\mathrm{S} 780}$ (Fig. 2d) in HCT116-PSLD 
Fig. 2 Signalling involving MET permits CDK2 activation in cells with CDK4/6 inhibition. a MetaCore ${ }^{\mathrm{TM}}$ GeneGO analysis identifies a signalling network engaging MET overrepresented by hits. Interaction types: $\mathrm{P}$, phosphorylation; B, binding; proteins targeted by a screen identified siRNA pools in blue.

b, c Hit validation using individual siRNAs (b) or pharmacological inhibitors for MET/MST1R (crizotinib or foretinib) or PTK2/2B (PDN1186 or defactinib) (c). Data depict SI score relating to loss of CDK2 activity in combination with CDK4/6 inhibiton, using palbociclib, determined using GFP-PSLD localisation. Data are mean \pm SD for $n=3$ independent repeats. $\mathbf{d}$, $\mathbf{e}$ Concentration-effect analysis depicting a change in the fraction of cells with low CDK4/ 6 activity, assessed using automated microscopy analysis of cells immunostained for pRB $1^{\mathrm{S} 780}(\mathbf{d})$ or low CDK2 activity, assessed based on GFPPSLD localisation (e) following individual or combined inhibition of CDK4/6, using palbociclib, and MET, using crizotinib. Data are mean \pm SD for three independent repeats. $* * * * p \leq 0.0001,{ }^{\mathrm{ns}} p>0.05$, oneway ANOVA comparing effects across concentrations against vehicle or comparing palbociclib alone against palbociclib plus crizotinib (d) and comparing palbociclib alone or crizotinib alone against palbociclib plus crizotinib (e). f CI value plot calculated from data in (e). $F_{\mathrm{a}}=$ fraction affected a

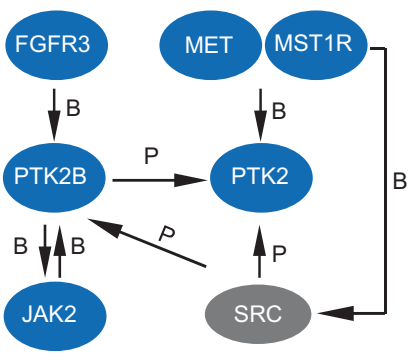

c

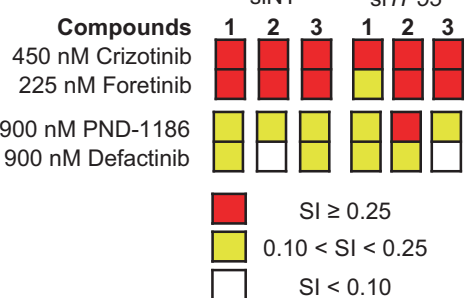

b
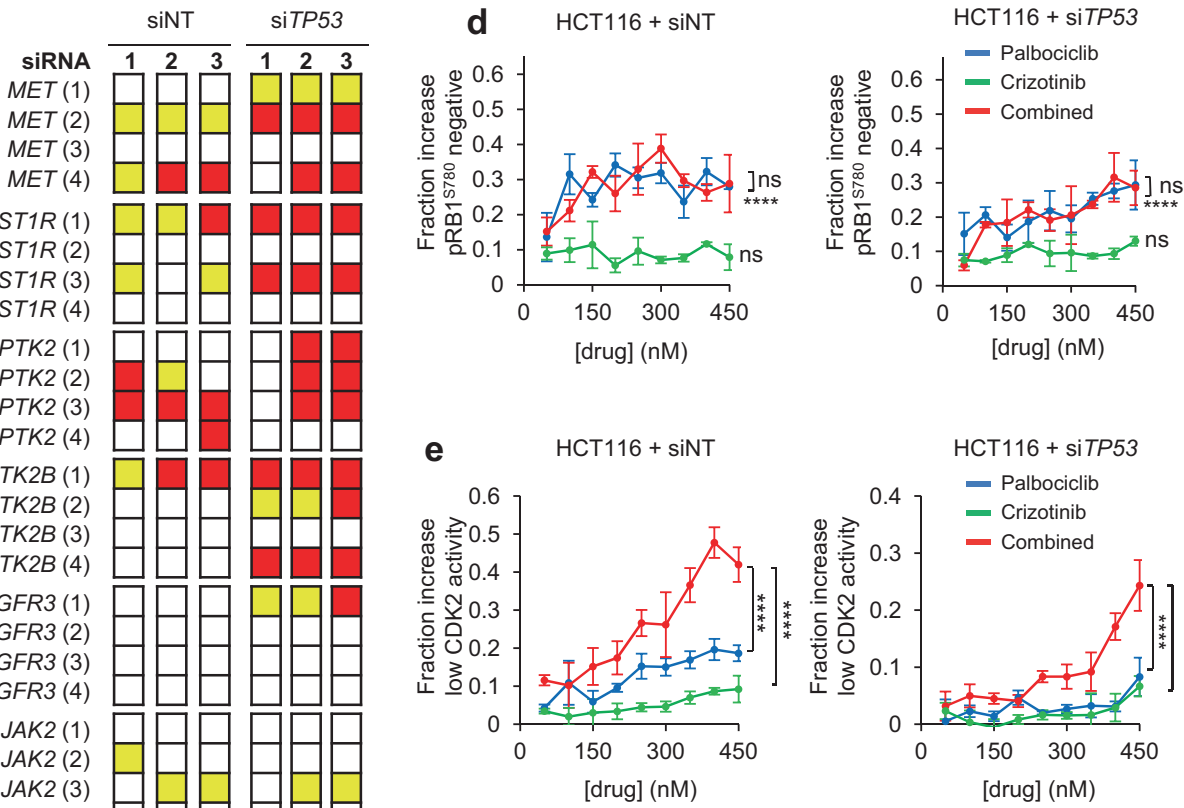

f

HCT116 + siNT

HCT116 + siTP53

$S R C(2)$

$S R C(3)$

$S R C(4)$

$\operatorname{MET}(1,4)$

$\operatorname{MST1R}(1,3)$

$M E T+M S T 1 R$

PTK2 $(2,3)$

$P T K 2 B(1,2)$

PTK2 + PTK2B

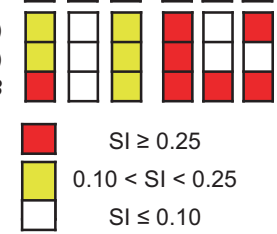

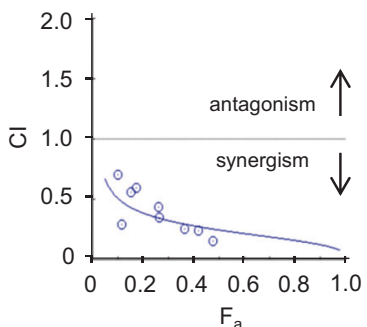

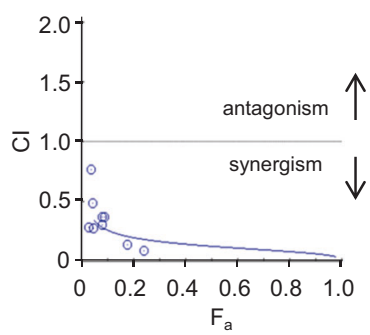

treated with individual and combined inhibitors. As expected, we observed a significant increase in the fraction of pRB1 ${ }^{\mathrm{S} 780}$-negative cells following CDK4/6 inhibition. Conversely, MET inhibition did not significantly increase the fraction of $\mathrm{pRB} 1^{\mathrm{S} 780}$-negative cells. Importantly, combined inhibition of CDK4/6 and MET was no more effective at raising the fraction of $\mathrm{pRB} 1^{\mathrm{S} 780}$-negative cells than inhibition of CDK4/6 alone at any concentration tested. Nevertheless, and in agreement with our earlier results, combined inhibition of CDK4/6 and MET led to a significantly greater reduction of cells with active CDK2 than treatment with either inhibitor alone (Fig. 2e). Chou-Talalay concentration-effect analysis [30] identified a robust synergistic interaction between MET and CDK4/6 inhibition towards reducing CDK2 activity, returning the combination index $(\mathrm{CI})$ values well below 1 across the concentration range tested (Fig. 2f), irrespective of TP53 status. Hence, MET inhibition cooperates with palbociclib to control CDK2 activation but does not enhance the ability of palbociclib to supress CDK4/6 activity. 


\section{Combined MET and CDK4/6 inhibition synergistically affects tumour cell fate in vitro and reduces tumour growth in vivo}

Since MET inhibition synergised with CDK4/6 inhibition to enable the control of CDK2, we tested if this treatment would also synergise to enable other responses associated with CDK4/6 inhibition. Inhibition of CDK4/6 is recognised for its ability to trigger permanent cell cycle exit, thought to underlie its anticancer activity [31]. To assess if MET inhibition enhances permanent cell cycle exit subject to CDK4/6 inhibition, we exposed cells for 5 consecutive days to inhibitors, then quantified their ability to form colonies by seeding equal numbers of live cells into the inhibitor-free medium (Fig. 3). We initially measured the response of HCT116, using two chemically unrelated inhibitors of CDK4/6, palbociclib and abemaciclib, and two chemically distinct inhibitors of MET family kinases, crizotinib and foretinib (Fig. 3a-d), in accordance with best practice [32].

Combinatorial treatment significantly enhanced the reduction in colony outgrowth compared with individual inhibitors $(p<0.01$, two-way ANOVA). Identical outcomes were obtained for HCT116 with genomic deletion of the TP53 gene $\left(\right.$ TP53 $\left.3^{-1-}\right)$ [33] or isogenic HCT116 with functional TP53 $\left(\right.$ TP53 $\left.{ }^{\mathrm{WT}}\right)$, and highly synergistic $(\mathrm{SI} \geq 0.25)$ or synergistic $(\mathrm{SI}>0.1)$ interactions were observed, regardless of inhibitor chemotype or combination partner (Fig. 3b-d). Biomarker analysis at $24 \mathrm{~h}$ confirmed that inhibitors appropriately modulated their respective targets (Fig. 3a). Thus, $\mathrm{pRB} 1^{\mathrm{S} 780}$ had decreased where CDK4/6is were used, while MET autophosphorylated on Tyr1234/1235 (pMET $^{\text {Y1234/5) }}$ had decreased in cells exposed to the METis. As noted previously, MET inhibition did not affect $\mathrm{pRB} 1^{\mathrm{S} 780}$ phosphorylation, nor did CDK4/6 inhibition affect the METactivation state. Cooperativity between MET inhibition and CDK4/6 inhibition in reducing colony-formation capability was also observed in cell lines derived from other cancer types, namely in the oestrogen/progesterone receptor-positive MCF7 human breast carcinoma-derived cells (MCF7) and in the KRAS-mutated A549 human lung adenocarcinomaderived cells (A549) (Fig. 3e, f).

Combined inhibition of MET and CDK4/6 also synergised towards loss of $\mathrm{Ki}-67$ expression (Fig. $4 \mathrm{a}-\mathrm{c}$ and Supplementary Fig. 2). Loss of Ki-67 is indicative of cell cycle exit [34] and predicts the response to CDK4/6 inhibition in preclinical models [7]. Combined inhibition of CDK4/6 and MET for 5 days significantly and cooperatively increased the percentage of HCT116 negative for Ki67, compared with treatment with single agents-irrespective of TP53 status (Fig. 4a)-yielding CI values well below 1 across the concentration range tested (Supplementary Fig. 2a).
Loss of Ki-67 in individual cells correlated with nuclear localisation of the GFP-PSLD reporter in the same cells (Supplementary Fig. 2b, c), supporting that reduction of CDK2 activity and loss of Ki-67 are mechanistically linked. Furthermore, different MET and CDK4/6i chemotypes synergised to reduce the fraction of Ki-67-positive cells within treated populations (Supplementary Fig. 2d), indicating that the observed effect is robust and involves ontarget MET and CDK4/6 inhibition.

Importantly, MET and CDK4/6 inhibition synergistically reduced $\mathrm{Ki}-67$ expression across a set of cancer cell lines, with diverse tissues-of-origin and genetic driver profiles (Fig. 4b, c). Concentration-effect analysis confirmed a synergistic interaction for 10 out of 12 lines tested. Notable exceptions were two RB1-mutated lines, the osteosarcoma line SAOS2 and the cervical cancer cell line C-33A, where combination treatment had additive or less than additive effects (Fig. 4b, c), consistent with the notion that RB1 is a critical downstream effector of CDK4/6 and that RB1 loss renders cells unresponsive to CDK4/6is. Thus, combined MET and CDK4/6 inhibition synergises broadly across cancer histio- and genotypes. The absence of synergism in RB1-mutated backgrounds identifies RB1 activity as a critical component required for this synergistic interaction.

We also assessed if combined inhibition of MET and CDK4/6 enhances or enables senescence by quantifying senescence-associated $\beta$-galactosidase ( $\mathrm{SA}-\beta$-gal) in cells. CDK4/6is induce cellular senescence, which is thought to underlie the loss of clonogenic activity they induce $[3,31,35]$. Using $C_{12} F D G$, a $\beta$-galactosidase substrate with green-fluorescent reaction product permitting automated quantitative analysis, we observed an overt increase in cells with $\mathrm{C}_{12}$ FDG fluorescence with characteristic distribution in the perinuclear region following treatment with the inhibitor combination (Supplementary Fig. 3a, b). Combinationtreated cells displayed additional changes associated with cellular senescence, including flattened shape, enlarged nuclei and increased cell size. Automated quantitative analysis confirmed a concentration-dependent increase in the fraction of cells with above-baseline perinuclear green fluorescence following combined, compared with singleagent treatment with METis and CDK4/6is (Fig. 4d), with concentration-effect analysis confirming a robust synergistic interaction for the expression of this senescence marker (Supplementary Fig. 3c).

Finally, we evaluated cell proliferation activity using cells modified to express the nuclear marker GFP-H2B to track division by time-lapse microscopy (Supplementary Fig. 3d-i). These experiments revealed a synergistic reduction in duplication activity subject to combined inhibition of MET and CDK4/6-irrespective of TP53 status.

Together, these results support the notion that MET inhibition synergistically increased known cellular responses 
Fig. 3 CDK4/6 and METis cooperate to incapacitate cell reproduction. a-d Clonogenic activity in HCT116 determined $120 \mathrm{~h}$ following inhibitor exposure to inhibitors of CDK4/ 6 (palbociclib or abermaciclib) or MET (crizotinib or foretinib). Data for HCT116 TP53 ${ }^{\mathrm{WT}}$ and $\mathrm{TP}^{-1-}$ are shown. Inhibitor response biomarkers $\mathrm{pRB} 1^{\mathrm{S} 780}$ (for CDK4/6 activity) and pMET $^{\text {Y1234/5 (for MET activity) }}$ (a), determined $48 \mathrm{~h}$ following inhibitor addition. GAPDH served as a loading control. b-f Clonogenic activity determined $120 \mathrm{~h}$ following inhibitor exposure in MCF7 and A549. Exemplary raw data $(\mathbf{b}, \mathbf{e})$, and quantification of clonogenic survival (mean $\pm \mathrm{SD}, n=3$ independent repeats) $(\mathbf{c}, \mathbf{d}, \mathbf{f})$ is shown. Palbociclib and crizotinib were used at $450 \mathrm{nM}$, abemaciclib and foretinib at $225 \mathrm{nM} .^{\dagger \dagger}$ : SI $\geq 0.25$ (highly synergistic), ${ }^{\dagger \dagger}: 0.25>\mathrm{SI} \geq 0.1$ (synergistic), $* * * * p \leq 0.0001$, one-way ANOVA a
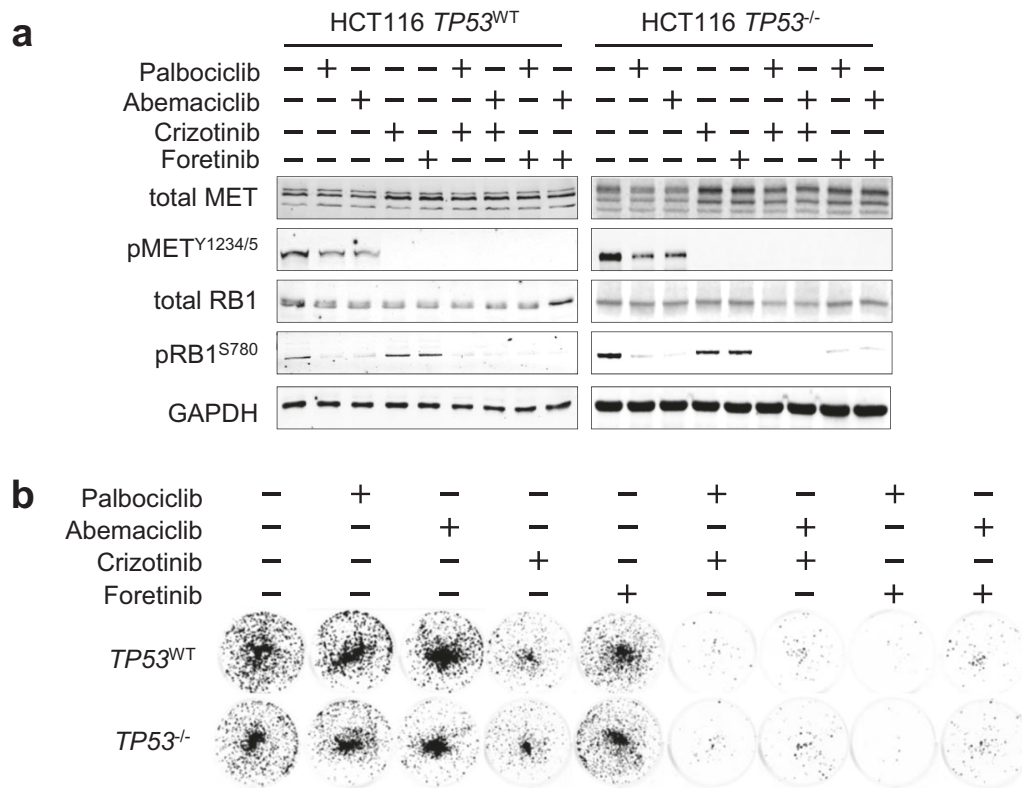

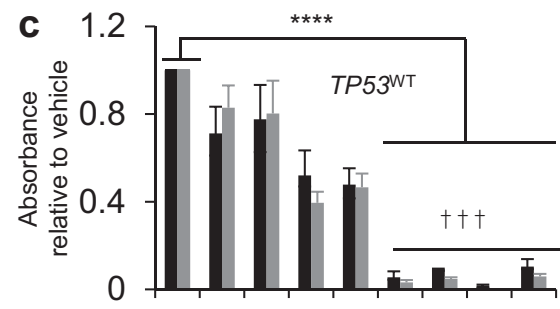

Palbociclib $-+-\quad-+-+-$ Abemaciclib - - + - - + + Crizotinib - - +-++-Foretinib ---++-+

Cells seeded:
$4000 \quad 1000$

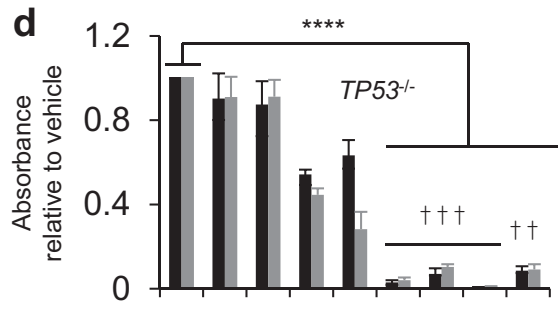

Palbociclib -+---+-+Abemaciclib -++--+-+ Crizotinib ---+-++Foretinib ---+-++ Cells seeded:
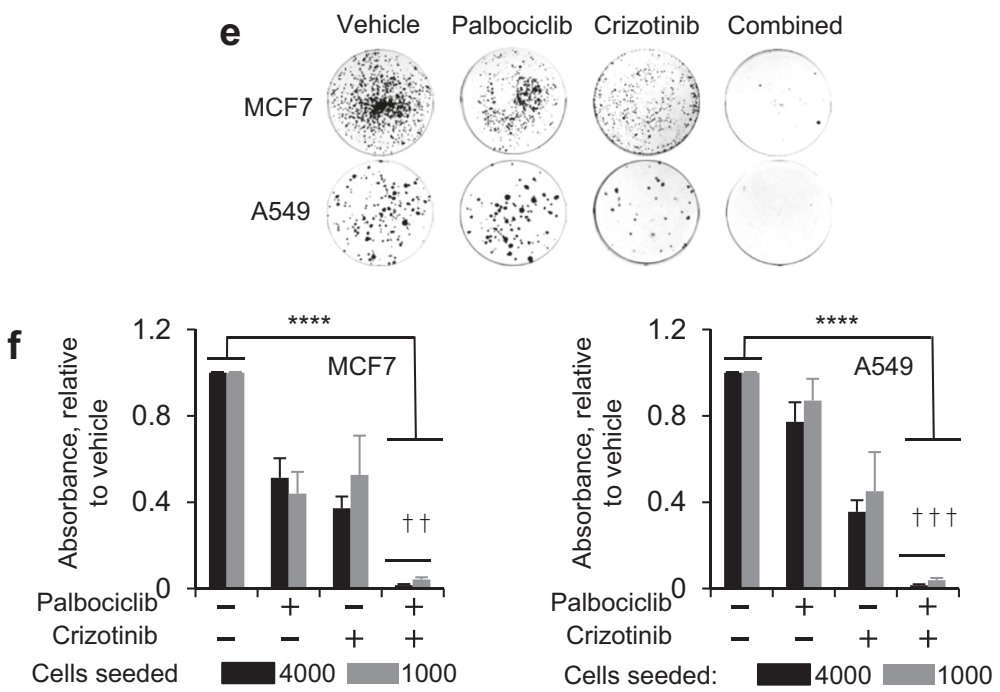

associated with CDK4/6 inhibition. Conversely, the results support a role for MET in preventing these responses in cells treated with CDK4/6is, including responses that predict antitumour response to CDK4/6 inhibition in vivo.
To test if CDK4/6 inhibition combined with MET inhibition is a feasible strategy for cancer treatment in vivo, we assessed the combined effect of crizotinib and palbociclib on the growth of human tumour xenografts in athymic mice. 
a

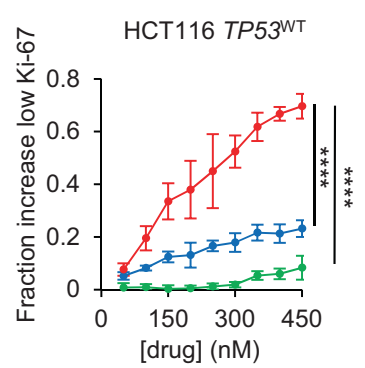

b

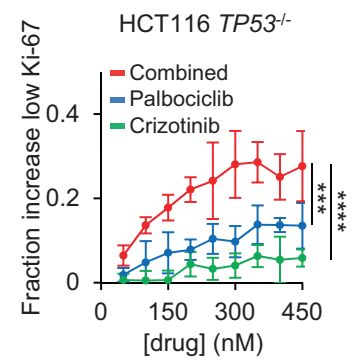

d

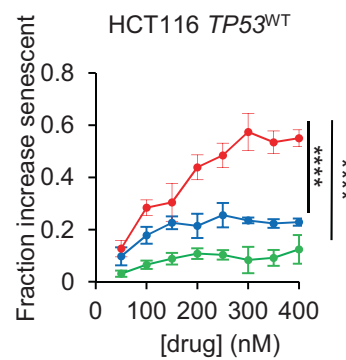

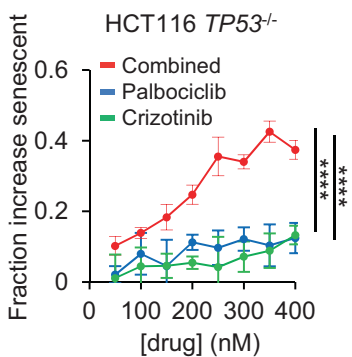

e

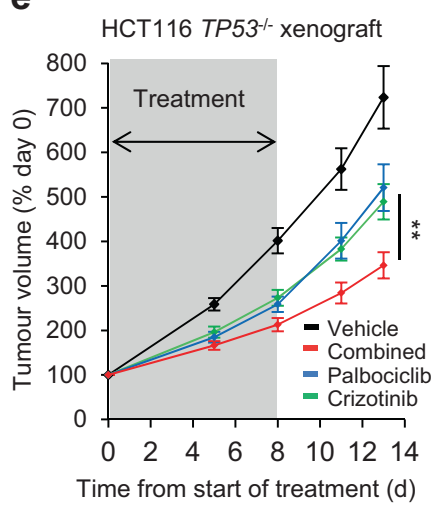

C
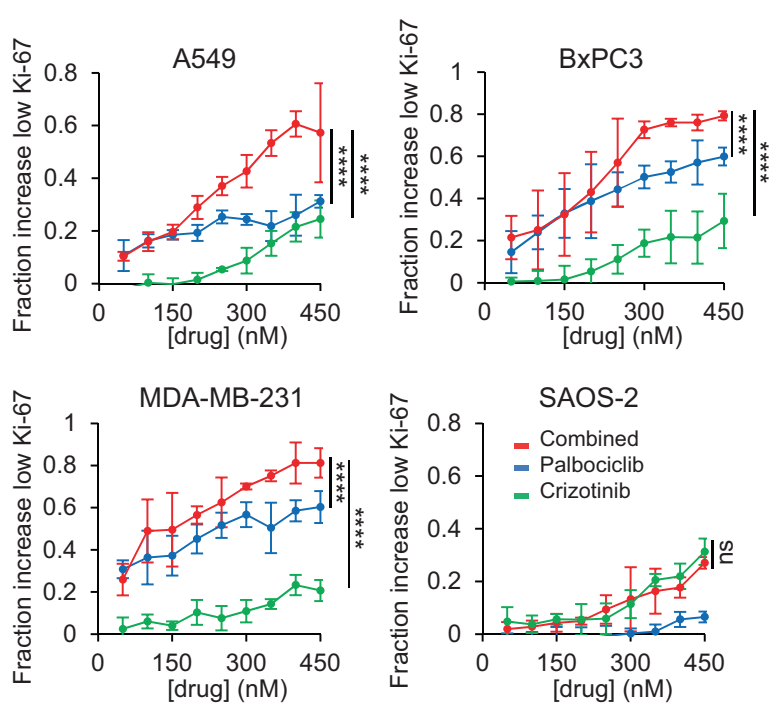

f
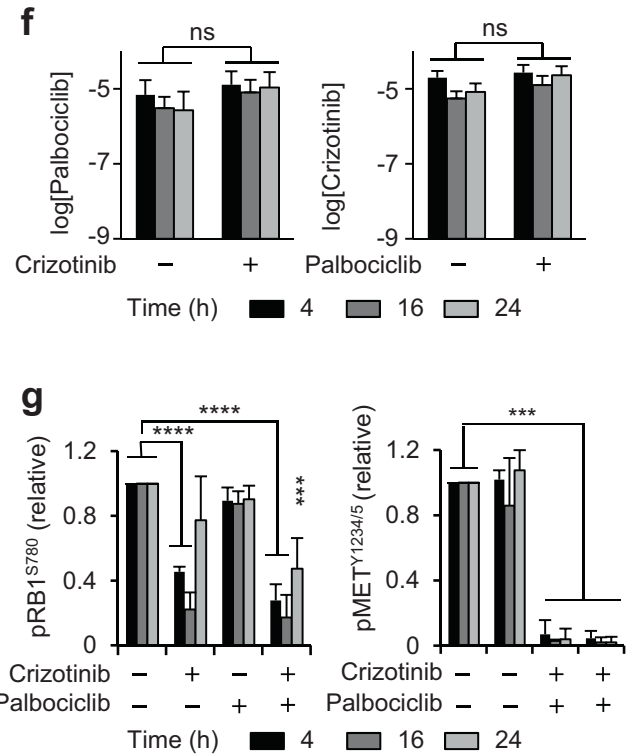

Combined daily administration of $100 \mathrm{mg} / \mathrm{kg}$ (p.o.) crizotinib and palbociclib was well tolerated in the mice (Supplementary Fig. 3k), yielding sustained accumulation of agents within tumour tissue (Fig. 4f) and modulation of $\mathrm{pRB} 1^{\mathrm{S} 780}$ and pMET ${ }^{\mathrm{Y} 1234 / 5}$ pharmacodynamic biomarkers over a 24 -h period (Fig. 4g).

Using this schedule, we treated mice bearing HCT116 $T P 53^{--}$tumour xenografts (Fig. 4e) with individual inhibitors or their combination for 8 days, then followed them for a further 5 days, when tumours in the control group reached predetermined size limits. This analysis confirmed superior efficacy of the combination, with statistically significant reduction in tumour burden compared with single-agent treatment at the end of the observation period ( $p=0.005$ against palbociclib and $p=0.006$ against crizotinib, Student's $t$ test). 
Fig. $4 \mathrm{MET}$ and CDK4/6is cooperate to promote cell cycle exit and tumour response in vivo. a Concentration-effect analysis depicting the fraction of HCT116 with low Ki-67, determined using automated microscopy analysis of cells stained with Ki-67 antibody MIB1. Cells were treated with inhibitors for $96 \mathrm{~h}$. Data represent means \pm SD for $n=3$ independent repeats. b, $\mathbf{c}$ Effect of CDK4/6 and MET inhibition on the Ki-67 labeling index across a cancer cell line panel. Cells were treated with palbociclib and/or crizotinib for $96 \mathrm{~h}$. CI value calculation (b) and exemplary results (c) based on two or more independent repeats run in duplicate each are shown. $\mathrm{CI}^{50}, \mathrm{CI}^{75}$ and $\mathrm{CI}^{90}$ denote $\mathrm{CI}$ values at concentrations with 50,75 or $90 \%$ of cells responding with Ki-67 loss, respectively, na $=$ fractional responses not achievable within the inhibitor concentration range tested. The mutation status of key oncogenic drivers is indicated for each cancer cell line. Data represent mean $\pm \mathrm{SD}$ for $n=2$ independent repeats, run in duplicate each. d Concentration-effect analysis depicting the fractional increase in cells with high $\mathrm{SA}-\beta$-gal assessed using automated microscopy of cells reacted with a fluorescencent substrate $\mathrm{C}_{12} \mathrm{FDG}$. Data represent mean $\pm \mathrm{SD}$ for $n=3$ independent repeats, run in triplicate each. e HCT116 tumour xenograft volumes, relative to day 0 , in control- and inhibitor-treated mice (mean $\pm \mathrm{SD}, n=10$ ). f Concentration of inhibitors in tumour xenograft tissue (mean $\pm \mathrm{SD}, n=3$ tumours). g Modulation of biomarkers indicative of CDK4/6 (pRB1 $\left.{ }^{\mathrm{S} 780}\right)$ and MET (pMET ${ }^{\mathrm{Y} 1234 / 5}$ ) activity in tumour xenograft tissue (mean $\pm \mathrm{SD}$, $n=3$ tumours). ${ }^{* *} p \leq 0.01, * * * p \leq 0.001$, and $* * * * p \leq 0.0001$; twoway ANOVA comparing the effect size of a single agent against that of the combination $(\mathbf{a}, \mathbf{c}, \mathbf{d})$ or two-sided unpaired Student's $t$ test $(\mathbf{e}, \mathbf{f}$, g). (Related to Supplementary Fig. 2 and Supplementary Fig. 3)

Together, these results provide evidence that MET activity constitutes a resistance mechanism in vitro and in vivo, reducing the tumour cell response to CDK4/6 inhibition. Importantly, our findings indicate that combined pharmacological inhibition of MET and CDK4/6 is a feasible strategy able to improve tumour growth inhibition in mice, indicating the potential of this combination for clinical use.

\section{Synergistic inhibition of CDK2 by MET and CDK4/6is involves $\mathrm{p} 21^{\mathrm{CIP} 1}$}

To identify the mechanism by which MET and CDK4/6 inhibition synergise, we characterised activity and composition of the CDK2 complex in inhibitor-treated cells using immunoprecipitation. These experiments confirmed a significant reduction in CDK2 activity subject to combined MET and CDK4/6 inhibition, determined by the ability of the anti-CDK2 immunoprecipitates to yield phosphorylation of substrate (GST-RB 763-928) in vitro (Fig. 5a, b, Supplementary Fig. 4a-d).

The decrease in CDK2 activity was not accompanied by a decrease in the amount of cyclin E1 (CCNE1) or a change in the phosphorylation of CDK2 at $\mathrm{Tyr} 15\left(\mathrm{pCDK} 2{ }^{\mathrm{Y} 15}\right)$ or Thr160 (pCDK2 ${ }^{\mathrm{T} 160}$ ), known to confer negative and positive regulation of CDK2 activity, respectively [36] (Fig. 5a). However, there was a significant increase in the amount of the CDK inhibitor protein (CDKI) $\mathrm{p} 21^{\mathrm{CIP} 1}$ in precipitates from cells treated with combined MET and CDK4/6i, as compared with single-agent- or vehicle-treated cells. Decreased CDK2 activity and the increased presence of $\mathrm{p} 21^{\mathrm{CIP} 1}$ in anti-CDK2 precipitates were consistently observed in multiple tumour cell lines subject to combined MET and CDK4 inhibition (Fig. 5a, c). Analysis of input lysates revealed loss of $\mathrm{pRB} 1^{\mathrm{S} 780}$ in samples with CDK4/6 inhibition and loss of $\mathrm{pMET}^{\mathrm{Y} 1234 / 5}$ in samples with MET inhibition (Fig. 5d), verifying single- agent activity and confirming that these proximal biomarkers and pathways are independently modulated by the respective inhibitors, regardless of cell background. Essentially identical results were obtained in immunoprecipitations performed, using an antibody for the CDK2-activating cyclin E (Supplementary Fig. 4e-m). Together, these results confirm that reduction of CDK2 activity is a cooperative event caused by combined inhibition of CDK4/6 and MET. They further identify binding of $\mathrm{p} 21^{\mathrm{CIP} 1}$ to the CDK2 complex as a potential cause underlying the cooperative interaction of these inhibitors.

To assess if the increased association of $\mathrm{p} 21^{\mathrm{CIP} 1}$ with the CDK2 complex links to increased $\mathrm{p} 21^{\mathrm{CIP} 1}$ abundance, we analysed lysate from treated cells using immunoblotting (Fig. 5d). A progressive increase in $\mathrm{p} 21^{\mathrm{CIP} 1}$ abundance was seen between 24 and $48 \mathrm{~h}$ subject to combination treatment (Fig. 5e, with quantification in Supplementary Fig. 5a). This increase in $\mathrm{p} 21^{\mathrm{CIP} 1}$ was apparent in TP53 $3^{-/}$HCT116, indicative that the upregulation of $\mathrm{p} 21^{\mathrm{CIP} 1}$, a known target transcriptionally activated by TP53, is TP53 independent. We also examined the abundance of the two other members of the CIP/KIP CDKI family, revealing an increase in $\mathrm{p} 27^{\mathrm{KIP} 1}$ at $48 \mathrm{~h}$, but not at earlier times. No change in the levels of p $57^{\mathrm{KIP} 2}$ was observed (Fig. 5e and Supplementary Fig. 5a).

We further assessed the level of the SKP1-cullin-F-box ubiquitin ligase substrate-recognition subunit (SKP2), involved in regulating the stability of CIP/KIP family proteins. A clear reduction in SKP2, detectable at $24 \mathrm{~h}$ and pronounced at $48 \mathrm{~h}$, was seen following combined inhibition of MET and CDK4/6 compared with vehicle-treated HCT116 (Fig. 5e and Supplementary Fig. 5a). The abundance of SKP2 was not affected by CDK4/6 or MET inhibition alone. Combined MET and CDK4/6 inhibition led to upregulation of $\mathrm{p} 21^{\mathrm{CIP} 1}$ and loss of SKP2 in multiple other cell backgrounds (Fig. 5e, with quantification in Supplementary Fig. 5b, 5c), indicative that this response is broadly observable. Together, these experiments indicate that signalling through MET and CDK4/6 acts redundantly to downregulate the CDKIs $\mathrm{p} 21^{\mathrm{CIP} 1}$ and $\mathrm{p} 27^{\mathrm{KIP} 1}$, and to upregulate their regulator SKP2, in turn providing a potential explanation why combined inhibition of MET and CDK4/6 is required for the inhibition of CDK2 and cellular proliferation capacity.

To evaluate if CIP/KIP family members are involved in the synergistic control of CDK2, we depleted these CDKIs alone or in combination using siRNA and assessed the 
a

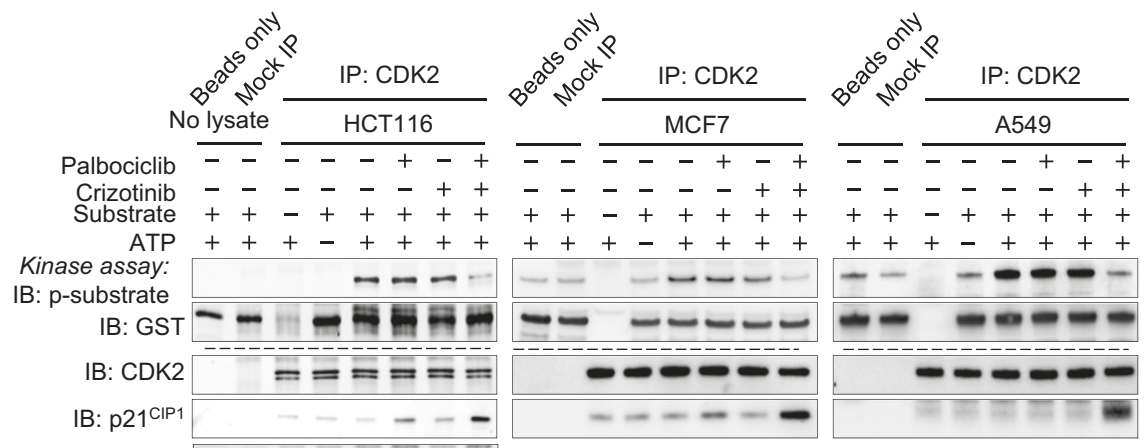

IB: CCNE1

IB: $\mathrm{pCDK} 2{ }^{\mathrm{Y} 15}$

IB: $p C D K 2^{\top 160}$

$\equiv=-\equiv=$

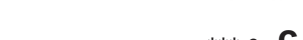
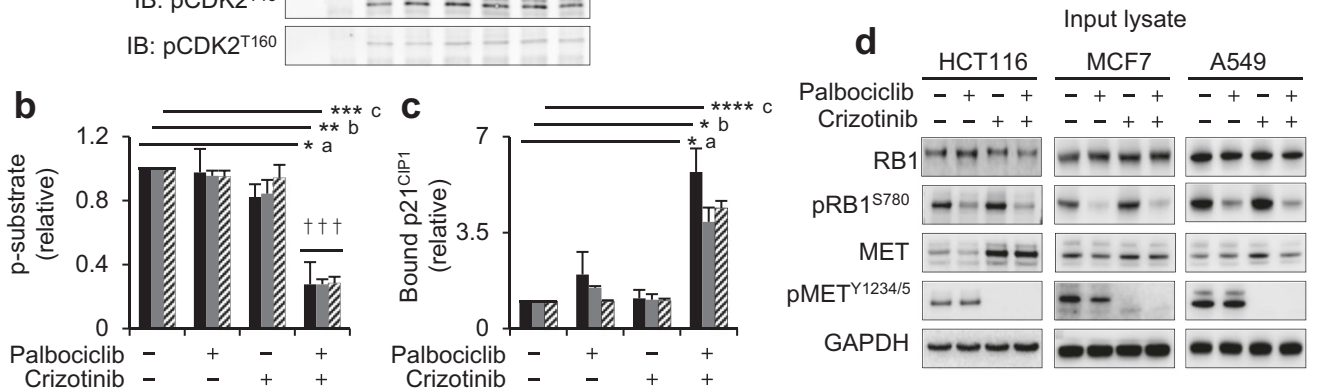

Palbociclib
Crizotinib

- HCT116 MCF7 ए20 A549

e
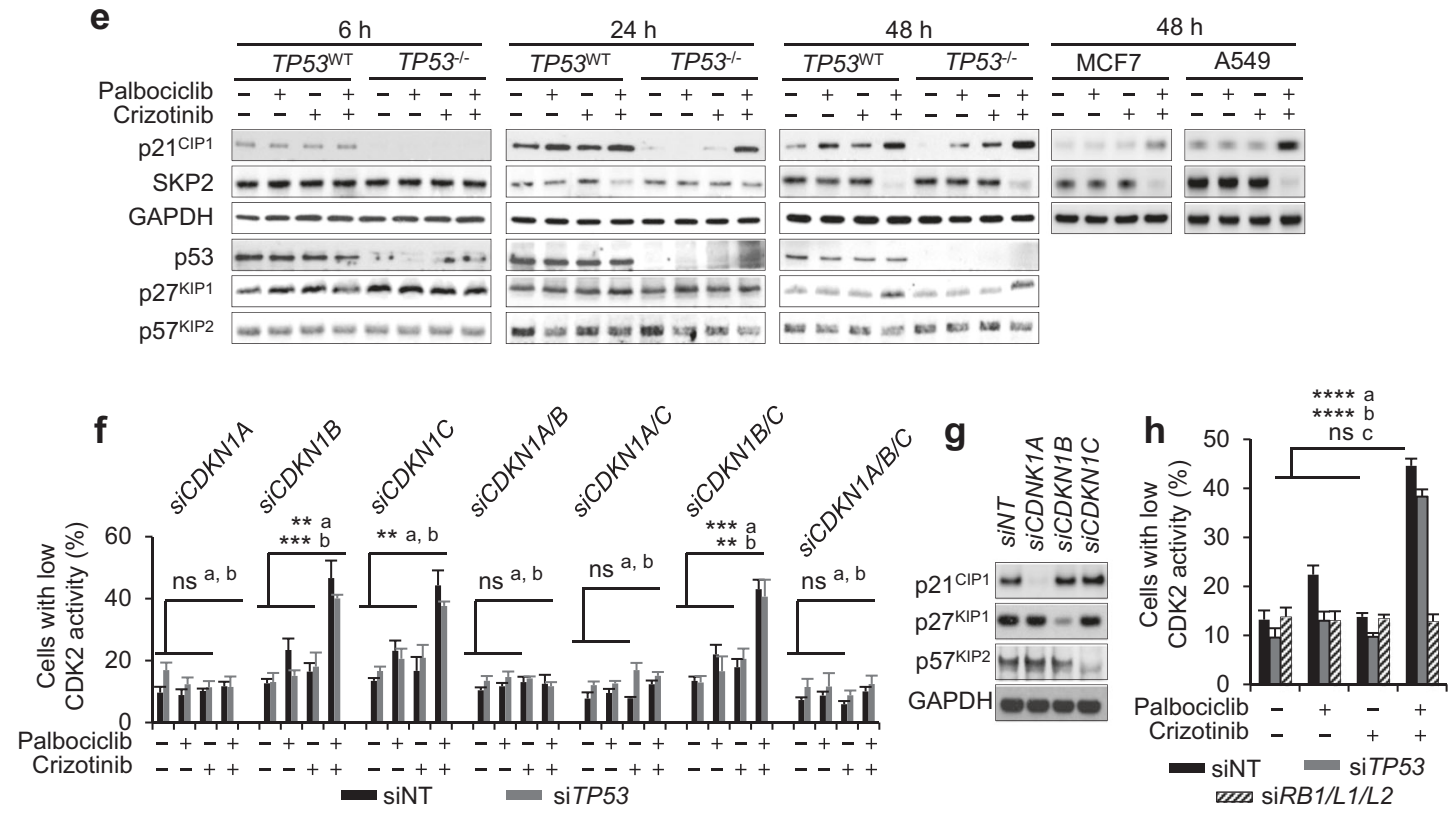

effect of this on CDK2 control, using the localisation of the GFP-PSLD reporter (Fig. 5f). These experiments positively identified $\mathrm{p} 21^{\mathrm{CIP} 1}$ as a critical mechanistic component in controlling CDK2 by combined MET- and CDK4/6 inhibition. Thus, transfection of cells with siRNA targeting CDKN1A (which encodes $\mathrm{p} 21^{\mathrm{CIP1}}$ ) consistently prevented CDK2 control by the combination. siRNA targeting $C D K N 1 B$ (encoding $\mathrm{p} 27^{\mathrm{KIPl}}$ ) and CDKN1C (encoding $\mathrm{p} 57^{\mathrm{KIP} 2}$ ) alone or in combination had no effect, despite evidence that the siRNAs effectively depleted the CDKIs concerned (Fig. 5g). Similar results were obtained in cells simultaneously transfected with TP53-targeted siRNA, indicating a critical role of $\mathrm{p} 21^{\mathrm{CIP} 1}$, regardless of TP53 status.

CDK2 regulation by the MET- and CDK4/6i combination was also prevented following loss of RB family proteins, RB1, RBL1 and RBL2 (Fig. 5h), consistent with the known resistance to CDK4/6is of cells with functional RB loss and indicative of a critical mechanistic role of $\mathrm{RB}$ protein function in the control of CDK2 by combined MET and CDK4/6 inhibition. 
Fig. 5 Cooperative control of CDK2 by MET and CDK4/6i involves p $21^{\mathrm{CIP} 1}$. a-d Characterisation of CDK2 complex using anti-CDK2 immunoprecipitation. Data for HCT116, MCF7 and A549 are shown. Cells were treated with $500 \mathrm{nM}$ of inhibitors for $24 \mathrm{~h}$ (HCT116) or $48 \mathrm{~h}$ (MCF7 and A549). Representative immunoprecipitation kinase assay (a), depicting in vitro-phosphorylated GST-pRB 763-928 substrate (p-substrate) reporting CDK2 activity, and total substrate (GST) (upper), and abundance of co-precipitated p21 ${ }^{\mathrm{CIP} 1}$, total and phosphorylated $\mathrm{CDK} 2\left(\mathrm{pCDK} 2^{\mathrm{Y} 15}, \mathrm{pCDK} 2^{\mathrm{T} 160}\right)$ and cyclin $\mathrm{E}$ (CCNE1) in the respective immunoprecipitations (lower). Quantification (mean $\pm \mathrm{SD}, n>2$ independent repeats) of p-substrate (b) and coprecipitated $\mathrm{p} 21^{\mathrm{CIP} 1}$ (c) relative to levels in vehicle-treated cells, and immunoblots of input lysate (d) assessing biomarkers pRB1 ${ }^{\mathrm{S} 780}$ (for CDK4/6 activity) and $\mathrm{pMET}^{\mathrm{Y} 1234 / 5}$ (for MET activity) for samples analysed in this figure. ${ }^{*} p \leq 0.05, * * p \leq 0.01, * * * p \leq 0.01$, and $* * * * p \leq$ 0.0001, two-way ANOVA assessing the effect size of a single agent with effect size of their combination, ${ }^{a} \mathrm{HCT} 116,{ }^{\mathrm{b}} \mathrm{MCF} 7$, and ${ }^{\mathrm{c}} \mathrm{A} 549$.

${ }^{\dagger \dagger} \mathrm{SI} \geq 0.25$ (highly synergistic) calculated using mean values. e Representative immunoblots assessing levels of CIP/KIP family CDKIs and SKP2 in cell lysates after treatment of cells with $500 \mathrm{nM}$ inhibitors. $\mathbf{f}$ CDK2 activity, assessed using GST-PSLD localisation, in HCT116 after transfection with siRNA targeting CIP/KIP family proteins with or without siRNA targeting TP53, followed by treatment with $500 \mathrm{nM}$ inhibitors for $24 \mathrm{~h}$ (mean $\pm \mathrm{SD}, n=3$ independent repeats). ${ }^{\mathrm{ns}} p>0.05, * * p \leq 0.01, * * * p \leq 0.001$, two-way ANOVA assessing the effect size of a single agent against the effect size of their combination. ${ }^{\mathrm{a}}$ Unperturbed (siNT), ${ }^{\mathrm{b}} \mathrm{TP} 53$-perturbed (siTP53). g Immunoblot documenting loss of CIP/KIP family proteins following transfection with siRNA. h CDK2 activity, assessed using GST-PSLD localisation, in HCT116 after transfection with siRNA targeting RB family proteins or TP53. Data depict mean \pm SD for three independent repeats, ${ }^{\mathrm{ns}} p>0.05$, $* * * p \leq 0.001$, two-way ANOVA assessing the effect size of a single-agent treatment against effect size of their combination. ${ }^{\mathrm{a}}$ Unperturbed (siNT), ${ }^{\mathrm{b}} \mathrm{TP} 53$-perturbed (siTP53), ${ }^{\mathrm{c}} \mathrm{RB}$ perturbed (siRB1/L1/L2). GAPDH (d, e, g) served as a loading control. (Related to Supplementary Fig. 4 and 5)

\section{Constitutive FAK activity abolishes cooperation between METis and CDK4/6is}

Our siRNA screen identified the FAK family kinases PTK2 and PTK2B, which are known MET effectors, as candidates that facilitate CDK4/6-independent CDK2 activation. Hence, we sought evidence if signalling through these kinases is involved in the CDK4/6-independent CDK2 activation by MET and whether additional MET-engaged signalling might play a role. MET and its close relative MST1R connect to multiple effector pathways, amongst them RAS/RAF/ERK, PI3K/AKT, SRC and STAT3, which they activate in addition to signal transduction involving the FAK/PTK family (Supplementary Fig. 6a).

While effectively blocking MET autophosphorylation within the catalytic region (pMET ${ }^{\mathrm{Y} 1234 / 5}$ ) and at the MET multifunctional docking site $\left(\mathrm{pMET}^{\mathrm{Y} 1349}\right)$ and, further, autophosphorylation of MST1R at Tyr1353 (pMST1R ${ }^{\mathrm{Y} 1353}$ ) (Supplementary Fig. 6b), treatment of HCT116 with METi alone or combined with CDK4/6i did not affect the activation state of RAS/ERK, PI3K/AKT, SRC and STAT3, indicated by unchanged levels of activated AKT
$\left(\mathrm{pAKT}^{\mathrm{S} 473}\right), \mathrm{ERK} 1 / 2\left(\mathrm{pERK}^{\mathrm{T} 202 / \mathrm{Y} 204}\right)$, SRC $\left(\mathrm{pSRC}^{\mathrm{Y} 416}\right)$ and STAT3 (pSTAT3 ${ }^{\mathrm{Y} 705}$ ), most likely due to activation of these pathways by signalling independent of MET. In contrast, a clear reduction was seen in modifications signifying activation of PTK2 (pPTK2 ${ }^{\mathrm{Y} 576 / 577}$ and $\mathrm{pPTK} 2{ }^{\mathrm{Y} 925}$ ) and PTK2B (pPTK2 ${ }^{\mathrm{Y} 402}$ ), documenting reduced signal transmission through these kinases following MET inhibition. Together, the results identify FAK family activity as critically dependent on MET in HCT116 and loss of FAK family activation as a key change resulting from MET/ MST1R inhibition.

To obtain evidence if FAK family activation through MET is involved in CDK4/6i-resistant CDK2 activation, we generated HCT116 cells expressing a membrane-targeted, constitutively active PTK2 variant, CD2-PTK2 (Fig. 6a). Expression of CD2-PTK2 did not affect the ability of MET inhibition to block MET receptor activity, or the ability of CDK4/6 inhibition to block RB1 phosphorylation (Fig. 6a). However, expression of CD2-PTK2 permitted sustained, METi-resistant PTK2 activation, indicated by the presence of pY576/577-phosphorylated CD2-PTK2 (Fig. 6a). Significantly, in cells expressing CD2-PTK2, the activity of CDK2 in anti-CDK2 immunoprecipitates was unaffected by combined inhibition of CDK4/6 and MET (Fig. 6b, c). Furthermore, the increased association of $\mathrm{p}^{2} 1^{\mathrm{ClP} 1}$ following combined CDK4/6 and MET inhibition was abolished (Fig. 6b, d and Supplementary Fig. 6c-f).

Notably, expression of CD2-FAK reduced the ability of combined CDK4/6 and MET inhibition to decrease colony formation in HCT116 cells (Fig. 6e, f), irrespective of TP53 status. It also reduced the increase in $\mathrm{p} 21^{\mathrm{CIP} 1}$ and the loss of SKP2, associated with combined MET and CDK4/6 inhibition (Fig. 6g and Supplementary Fig. 6g). Together, these results indicate that reduction of FAK activity is key to limiting CDK2 and clonogenic activity following from combined MET and CDK4/6 inhibition in cells.

The finding that combined inhibition of the MET/FAK and CDK4/6 axis can promote $\mathrm{p} 21^{\mathrm{CIP} 1}$ accumulation in HCT116 TP53 $3^{--}$implies a mechanism for TP53-independent generation of $\mathrm{p} 21^{\mathrm{CIP} 1}$, conferred by inhibition of the MET/FAK axis. Consistent with this, and regardless of TP53 status, we observed a significant increase in CDKN1A mRNA (encoding p2 $1^{\mathrm{CIP1}}$ ) following combined CDK4/6 and MET inhibition compared with control or CDK4/6i-treated cells (Fig. 6h), which was abolished by the expression of constitutively active PTK2. Notably, the level of CDKNIA mRNA increased in cells treated with METi alone, indicating that CDKN1A transcription is suppressed by signalling through MET/FAK, independent of CDK4/6. Together, these experiments identify FAK family activity as a critical effector downstream of MET involved in preserving CDK2 activity and clonogenic potential in cells exposed to CDK4/6i. They further suggest 
a

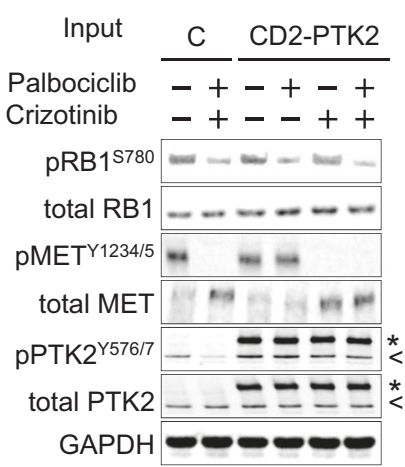

b

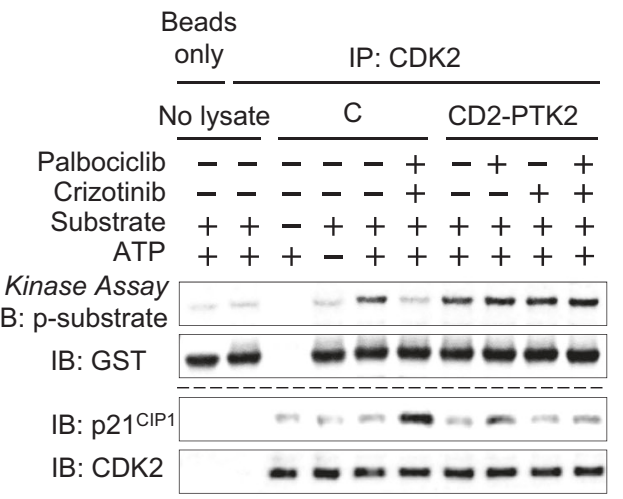

e

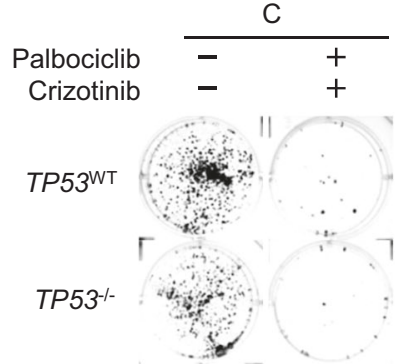

TP53 ${ }^{W T}$

f

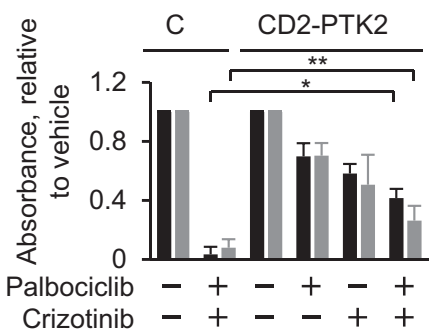

g
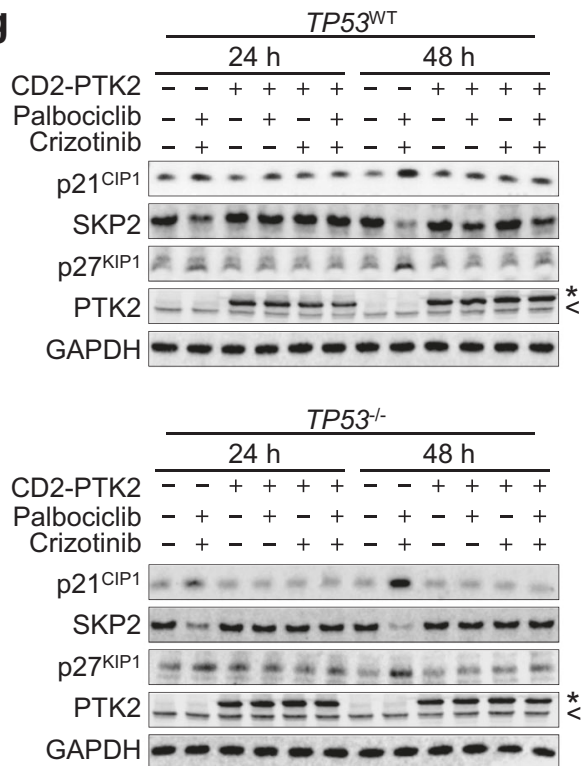

TP53\%
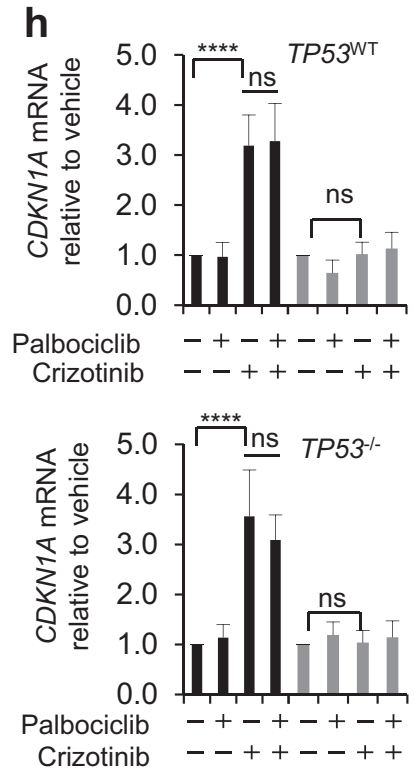

C $\quad \mathrm{C}$

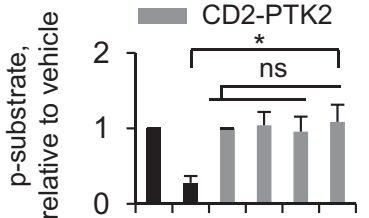

Palbociclib -+-+-+

Crizotinib -+--++

d

- C

CD2-PTK2

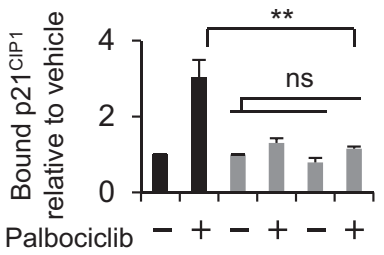

Palbociclib -+-+-+
Crizotinib -+--++

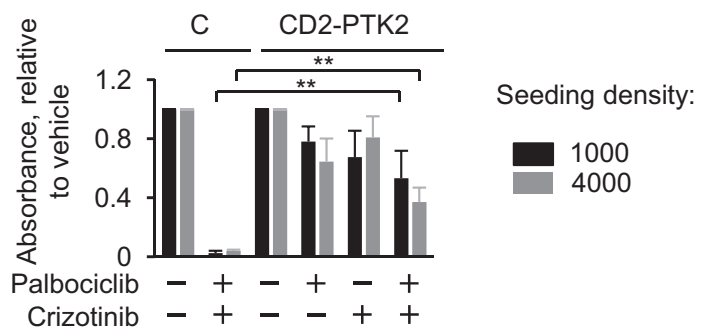

$C$

CD2-PTK2 
Fig. 6 Constitutive PTK2 activity impairs cooperation between MET and CDK4/6is. a-d Characterisation of CDK2 complexes in HCT116 expressing constitutively active CD2-PTK2. a Immunoblot of input lysate documenting CD2-PTK2 expression and modulation of drug response biomarkers $\mathrm{pRB} 1^{\mathrm{S} 780}$ (for CDK4/6 activity) and $\mathrm{pMET}^{\mathrm{Y} 1234 / 5}$ (for MET activity). C denotes control cells without CD2-PTK2 expression, <identifies a signal for cell-intrinsic PTK2, * identifies CD2-PTK2. b Representative immunoprecipitation kinase assay. Abundance of in vitro-phosphorylated GST-pRB 763-928 substrate (psubstrate) reflecting CDK2 activity and total substrate (upper), abundance of $\mathrm{p} 21^{\mathrm{CIP} 1}$ and CDK2 (lower) in the respective immunoprecipitations. $\mathbf{c}$ Mean abundance of p-substrate and $\mathbf{d}$ mean abundance of co-precipitated $\mathrm{p} 21^{\mathrm{CIP} 1}$ relative to vehicle-treated cells. Data (c, d) represent mean \pm range for two independent repeats. Cells were treated for $24 \mathrm{~h}$ using $500 \mathrm{nM}$ of each inhibitor. ${ }^{*} p \leq 0.05$, ${ }^{*} p \leq 0.01$, twoway ANOVA assessing the effect size of combination treatment between control and CD2-PTK2-expressing cells and ${ }^{\mathrm{ns}} p>0.05$, twoway ANOVA assessing the effect size of a single agent against effect size of their combination in cells expressing CD-PTK2. e-f Clonogenic survival of cells expressing constitutively active CD2-PTK2. e Exemplary raw images and $\mathbf{f}$ quantitative assessment representing mean values \pm SD for three independent repeats. Cells were treated with $500 \mathrm{nM}$ inhibitors for $120 \mathrm{~h}$. $* p \leq 0.05$, ** $p \leq 0.01$, two-way ANOVA assessing effect size of combination treatment between controls and CD2-PTK2-expressing cells. g Representative immunoblots assessing the abundance of CIP/KIP family proteins and SKP2 in HCT116, with constitutively active CD2-PTK2. Cells were treated with $500 \mathrm{nM}$ inhibitors. h RT/qPCR assessing CDKN1A mRNA levels. Cells were treated with $500 \mathrm{nM}$ inhibitors for $24 \mathrm{~h}$. Data are mean values $\pm \mathrm{SD}$ of three independent repeats; $* * * * p \leq 0.0001,{ }^{\mathrm{ns}} p>$ 0.05 , one-way ANOVA. GAPDH $(\mathbf{a}, \mathbf{g})$ served as a loading control. (Related to Supplementary Fig. 6)

TP53-independent $C D K N 1 A$ transcript accumulation as a candidate mechanism, by which inhibition of MET/FAK promotes CDK4/6i-resistant CDK2 activation and cell cycle activity.

\section{SKP2 is the critical common target engaged by MET and CDK4/6}

In addition to the increase in $\mathrm{p} 21^{\mathrm{CIP} 1}$, our earlier results showed a loss of SKP2 following combined CDK4/6 and MET inhibition (Fig. 5e and Supplementary Fig. 5). SKP2 mediates the degradation of $\mathrm{p} 21^{\mathrm{CIP} 1}$ [37] and itself is targeted for degradation involving $\operatorname{RB} 1[15,16]$. Therefore, SKP2 loss may be the consequence of sustained RB1 activity, following from effective control of CDK2 activity through $\mathrm{p} 21^{\mathrm{CIP} 1}$. SKP2 loss may stabilise p21 ${ }^{\mathrm{CIP} 1}$, synthesised from a transcript that accumulates as a consequence of inhibition of the MET/FAK axis.

To test if this model explains how METis and CDK4/6is synergise, we used siRNA or inhibitors to ablate the relevant components and assessed the effect of this on SKP2 loss and $\mathrm{p} 21^{\mathrm{CIP} 1}$ accumulation (Fig. 7). Consistent with the model, ablation of RB family proteins abolished SKP2 loss and $\mathrm{p} 21^{\mathrm{CIP} 1}$ accumulation, following combined MET and CDK4/6 inhibition (Fig. 7a, with quantification in
Supplementary Fig. 7a). Also, consistent with this model, PTK2 ablation, or pharmacological inhibition of FAK family activity using defactinib, was sufficient to trigger $\mathrm{p} 21^{\mathrm{CIP} 1}$ accumulation but insufficient to cause SKP2 loss, for which additional inhibition of $\mathrm{CDK} 4 / 6$ was required (Fig. 7b, quantification in Supplementary Fig. 7b).

Unexpectedly, SKP2 loss-predicted to require MET inhibition in order to yield upregulation of $\mathrm{p} 21^{\mathrm{CIP} 1}$-yielded $\mathrm{p} 21^{\mathrm{CIP} 1}$ accumulation that was not further enhanced by MET inhibition (Fig. 7c, quantification in Supplementary Fig. 7c). This result indicates that $\mathrm{p} 21^{\mathrm{CIP} 1}$ transcript upregulation caused by MET inhibition may not be essential for p $21^{\mathrm{CIP} 1}$ accumulation, at least under conditions where SKP2 is absent at the outset, and could indicate that MET/FAK inhibition has involvement in promoting SKP2 loss, independent from SKP2 degradation that it may enable by cooperating with CDK4/6 inhibition towards RB1 activation. Consistent with this prediction, we found that MET inhibition, further to increasing transcript levels for $\mathrm{p} 21^{\mathrm{CIP} 1}$, suppressed transcript levels for SKP2, which like the increase in the $\mathrm{p} 21^{\mathrm{CIP} 1}$ transcript, was counteracted by constitutively active PTK2 (Fig. 7d). Also consistent with this prediction, we found that SKP2 loss enabled palbociclib to control CDK2 activation without additional MET inhibition (Supplementary Fig. 7d).

Together, these data support a mechanistic model (Fig. 7e) in which SKP2 acts as a common target engaged by MET and CDK4/6 and proposes FAK-driven SKP2 and potentially $\mathrm{p} 21^{\mathrm{CIP} 1}$ transcript regulation as mechanistic events through which the MET/FAK axis confers a refractory response to $\mathrm{CDK} 4 / 6$ inhibition, and through which MET inhibition synergises with CDK4/6 inhibition to deliver increased anticancer activity.

\section{Discussion}

Widespread recognition exists that CDK2 activation is associated with acquired resistance of cancer cells to CDK4/ 6-targeting cancer therapeutics [21-23] and also that CDK2 supports CDK4/6-independent proliferation during organismal development [20]. However, the molecular determinants that permit activation of CDK2 in these contexts have not been systematically sought. CDK4/6-selective inhibitors are now showing considerable promise in patients with oestrogen receptor-positive breast cancer, yet there is clear need to identify functions that drive resistance in treatments involving these agents and that preclude their broader use in other cancer types. Here, we report that activation of the MET/FAK signalling axis leads to CDK4/ 6-independent CDK2 activation, and constitutes a broadly applicable druggable means to improve the response of cancers to CDK4/6-targeted therapies. 
Fig. 7 SKP2 is the critical function engaged by MET and CDK4/6. a-c Representative immunoblots assessing levels of $\mathrm{p} 21^{\mathrm{CIP} 1}, \mathrm{p} 27^{\mathrm{KIP} 1}$ and SKP2 protein in cells treated with siRNA against $R B$ family proteins (RB) (a), siRNA against PTK2 or the FAK inhibitor defactinib $(900 \mathrm{nM})(\mathbf{b})$ or siRNA targeting $S K P 2$ (c). Data for $T P 53^{\mathrm{WT}}$ and $T P 53^{-1-}$ HCT116, MCF7 and A549 are shown. Cells were treated with $500 \mathrm{nM}$ METi crizotinib and/or CDK4/6i palbociclib. GAPDH served as a loading control. d RT/qPCR assessing CDKN1A mRNA in HCT116 treated with $500 \mathrm{nM}$ inhibitors for $24 \mathrm{~h}$. Data are mean values \pm SD for three independent repeats; $* * p \leq 0.01$, one-way ANOVA comparing the effect of vehicle vs. treatment. e Model, detailing the consequence of combined CDK4/6- and MET inhibition. Grey box indicates an active node and red indicates active signalling. (Related to Supplementary Fig. 7) a

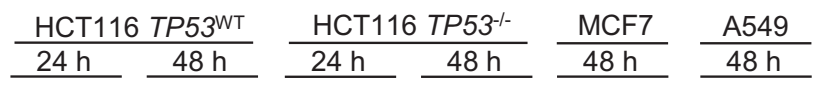

siRNA NT RB NT RB NT RB NT RB NT RB NT RB

Palbociclib $\overline{-+} \overline{-+-+} \frac{\mathrm{RB}}{-+} \overline{-+-+} \frac{\mathrm{RB}}{-+} \overline{\mathrm{RB}} \frac{\mathrm{NT}}{-+-+} \overline{\mathrm{RB}} \frac{\mathrm{NT}}{-+-+} \overline{\mathrm{RB}} \frac{\mathrm{NT}}{-++-+} \overline{\mathrm{RB}}$

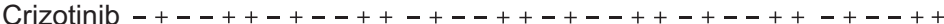

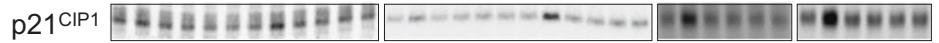

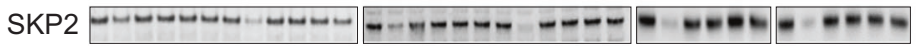

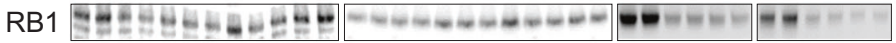

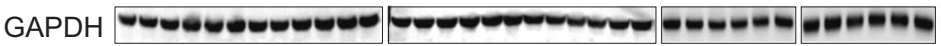

p27 KIP1 $--\quad \ldots+\cdots$

b

$\frac{\mathrm{HCT}_{116} \text { TP53 }}{24 \mathrm{hT}} \frac{\mathrm{HCT} 116 \mathrm{TP} 53^{-/-}}{24 \mathrm{~h} \quad 48 \mathrm{~h}}$

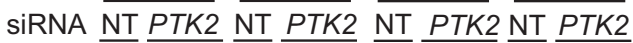

Palbociclib-+ $\overline{-+-+} \frac{\mathrm{P}}{-++-+} \frac{\mathrm{PTK}}{-++} \overline{-+-+} \frac{\mathrm{PTK}}{-+} \overline{-+-+}$

Crizotinib-+- - ++-+--++ - +- - ++- +- - ++

p21 CIP1 - -

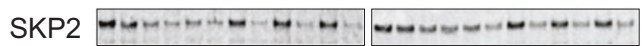

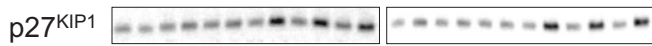

PTK2

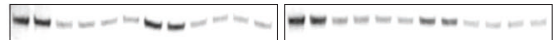

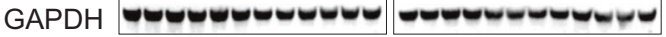

$\frac{\frac{\mathrm{MCF} 7}{48 \mathrm{~h}}}{\frac{\mathrm{A} 549}{48 \mathrm{~h}}}$

Palbociclib - +- +-+ - +-+-+

Crizotinib - + - - ++ - + - + +

p21 ${ }^{\mathrm{ClP} 1} \| \cdots \cdots \cdots * \cdots \cdots \cdots$

SKP2 $\bullet \bullet \bullet \bullet-m+m=\bullet$

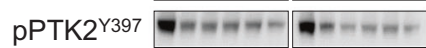

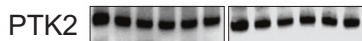

GAPDH - - - - - - - -

C

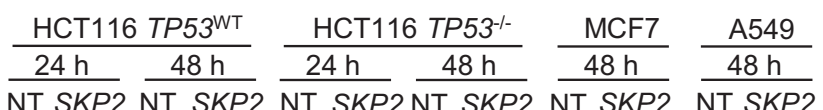

SiRNA $\overline{\text { NT SKP2 }} \overline{\text { NT SKP2 }} \overline{\text { NT SKP2 }} \overline{\text { NT SKP2 }} \overline{\text { NT SKP2 }} \overline{\text { NT SKP2 }}$

Palbociclib $\frac{N T}{-+++-+} \frac{\text { SKP }}{-+-+-+} \frac{\text { SKP }}{-++} \frac{\mathrm{NKP}}{-+-+} \frac{\mathrm{NT}}{-+++-+} \frac{\mathrm{SKP}}{-++} \frac{\mathrm{NT}}{-+-+} \frac{\mathrm{ST}}{-+++-+}$

Crizotinib - + - + + - +- - + + - + - - + +- +- - + + - + - - + + - + - + + +

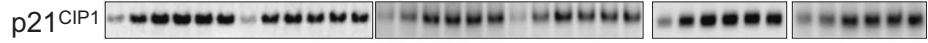

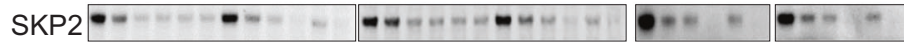

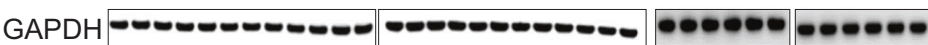

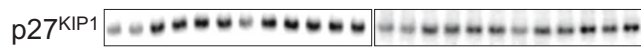

d

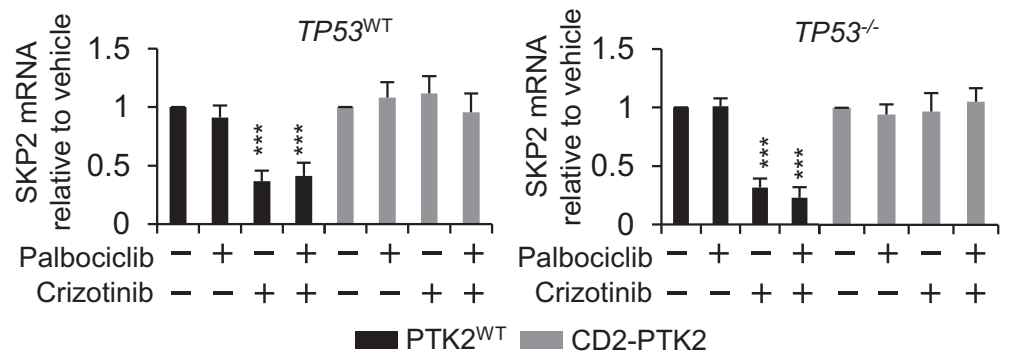

e

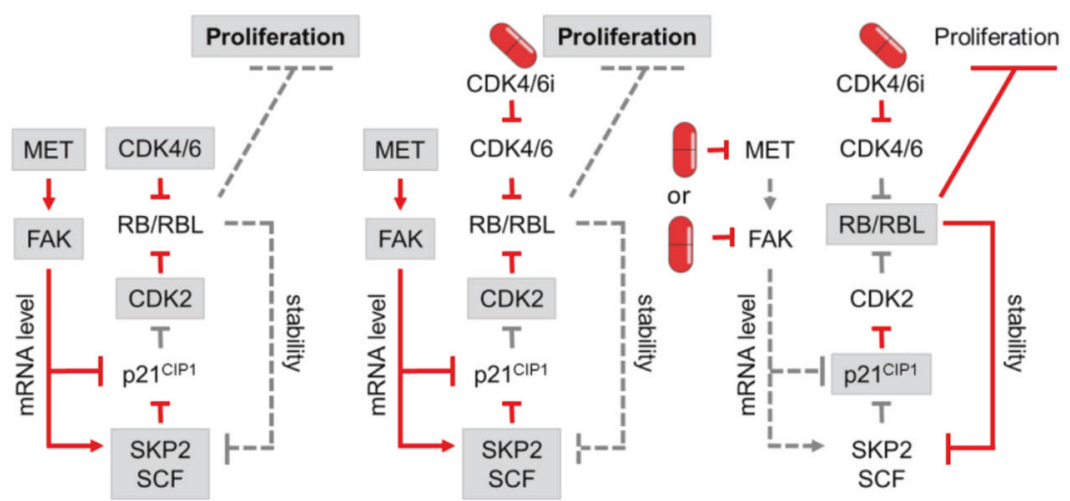


MET is widely expressed in epithelial and endothelial cells, including cancers derived from these tissues, and may be activated in cancer cells through mutation. However, more frequently, MET signalling is activated by the hepatocyte growth factor/scatter factor, HGF, produced by adjacent mesenchymal tissues, including stromal components of cancers [38-40].

We identified the MET/FAK axis based on a functional genetic screen, using CDK2 activity as a mechanism-based endpoint. Apart from the MET/FAK axis, which we mechanistically explore in our work, the screen yielded other hits without known links to MET and/or FAK signalling, including ontology noted previously for synergistic interaction with catalytic inhibition of CDK4/6, such as RPS6KA6 and BRAF, inhibitors of which increase the response of cancer cells to CDK4/6 inhibition [2, 21, 41]. Unexpectedly, our screen indicates that TP53 status affects the spectrum of molecular functions required to support CDK4/6-independent CDK2 activation, with requirement of certain functions, including, e.g. RPS6KA6, confined to TP53-normal backgrounds. While further validation is required, these observations suggest that the TP53 status could determine the efficacy of certain combinations.

In addition to MET, the screen identified FAK family kinases, that our subsequent work validates as a critical component by which MET elicits CDK4/6-independent CDK2 activation. Our experiments involving a constitutively active form of the FAK family kinase PTK2 demonstrate that FAK activity is sufficient to elicit significant CDK4/6i tolerance. In the experimental models examined, MET scores as a key determinant responsible for FAK family activation, as indicated by the robust reduction of activated FAK species pPTK2 ${ }^{\mathrm{Y} 576 / 577}$, pPTK2 ${ }^{\mathrm{Y} 925}$ and

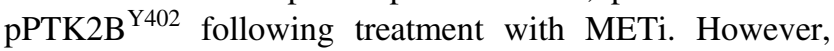
FAK family kinases can be activated by other routesincluding by MET-unrelated tyrosine kinase receptors and their activators, and by extracellular matrix signalling involving integrins [42]. The broad range of signalling able to engage FAK highlights the possibility that CDK4/6i resistance involving FAK could be caused in cancer patients by events other than MET activation.

Our work indicates that sustained expression of the ubiquitin ligase subunit SKP2, which promotes the degradation of $\mathrm{p} 21^{\mathrm{CIP} 1}$ as well as that of other CIP/KIP CDKIs, is a key mechanism by which MET/FAK supports activation of CDK2. Our experiments show that signalling through this axis increases the steady-state level of SKP2 mRNA. The involvement of FAK in the regulation of SKP2 and p $21^{\mathrm{CIP} 1}$ was previously noted. For example, inhibition of FAK through enforced expression of FAK-related nonkinase (FRNK) or dominant negative FAK (Tyr397Phe) reduced the expression of SKP2 protein in human and rat endothelial cells $[43,44]$. Furthermore, inhibition of FAK by FRNK, FAK (Tyr397Phe) or small-molecule inhibitors resulted in elevated $\mathrm{p} 21^{\mathrm{CIP} 1}$ transcript and protein in normal human fibroblasts, smooth muscle cells or glioblastomaderived cells $[43,45,46]$. These results independently support our observations that FAK family kinases regulate SKP2 and the CIP/KIP inhibitor $\mathrm{p} 21^{\mathrm{CIP} 1}$. How MET/FAK signalling affects SKP2 transcript levels is currently not known, but given the involvement of this signalling axis in CDK4/6i resistance, this is clearly an important aspect to be addressed in future studies.

Our work indicates that MET inhibition significantly enhances therapeutic inhibition of growth by CDK4/6is in preclinical models of human cancer. Furthermore, our results identify FAKs as key downstream components in this context. The recognised ability of cancer-relevant, MET-independent signalling routes to activate FAKs make this kinase family, or druggable targets downstream of it, potentially more attractive therapeutic targets than MET for use in combination with CDK4/6 inhibition. FAK family kinases are a recognised drug target in cancer, and inhibitors targeted to the ATP-binding pocket of their kinase domain have entered clinical trials, albeit with limited single-agent efficacy in patients thus far [47-49]. Our results suggest an opportunity for use of FAK inhibitors in conjunction with CDK4/6is as a potentially powerful approach to improve the outcome for patients treated with CDK4/6-targeted therapies or to expand the current, approved indications through mechanism-based targeted combinations.

\section{Methods}

\section{Cell culture, chemicals and antibodies}

HCT116 TP53 ${ }^{--}$and isogenic HCT116 TP53 ${ }^{\text {wt }}$ cells were provided by the Vogelstein laboratory (John Hopkins University, Baltimore, MD). Other cell lines were acquired from the American Type Culture Collection (ATCC). HCT116-PSLD are described in ref. [26]. Cells expressing CD2-PTK2 were constructed by lentiviral transduction using pLV-neo-CD2-FAK [50]. Inhibitors used were purchased from Selleck Chemicals. Antibodies and siRNAs used are summarised in supplementary materials.

\section{RNAi screens}

Screens used the kinase-covering component of the Dharmacon siGENOME SMARTpool ${ }^{\mathrm{TM}}$ library. Library pools were mixed at an equimolar ratio with SMARTpool ${ }^{\mathrm{TM}}$ oligonucleotides targeting TP53 or non-targeting oligonucleotide, then reverse transfected at a combined concentration of $20 \mathrm{nM}$ into HCT116-PSLD, seeded in 96-well plates. Transfected cells were incubated for $24 \mathrm{~h}$ prior to 
treatment with CDK4/6i palbociclib $(450 \mathrm{nM})$ or vehicle for $24 \mathrm{~h}$. Plates were fixed in $4 \%$ formaldehyde, then stained with Hoechst 33342 DNA dye and imaged using an INCell Analyzer 3000 (GE Healthcare) or an Opera (Perkin-Elmer) high-content imager platform. Data for a minimum of 2000 cells per condition were collected. Data were processed using CellProfiler open-source image analysis software [51].

\section{In vivo human tumour xenograft studies}

All animal work was carried out under UK Home Office regulations in accordance with the Animals (Scientific Procedures) Act 1986 and according to United Kingdom Co-ordinating Committee on Cancer Research guidelines for animal experimentation [52] with local ethical approval. For therapy studies, mice were treated daily for a continuous period, followed by observation, until tumour size in the control group approached predetermined humane size limits. For pharmacodynamic studies, tumour samples were collected at $24 \mathrm{~h}$ post administration.

Further method details are provided in the supplementary text.

Acknowledgements The authors thank Nicky Evans for editorial help with writing this paper, and Albert Hallsworth, Melanie Valenti and Gary Box for assisting with the mouse work. We thank the Vogelstein laboratory for providing isogenic $T P 53^{--}$and TP53 ${ }^{\mathrm{WT}}$ HCT116 human colorectal cancer cells. Financial support: The work was supported by grants from Cancer Research UK (ref. C309/A11566, ref. C309/A8274 and ref. C309/A8992 (PW), ref. C423/A1421 and ref. C423/A15043 (SM)) and the World Cancer Research Fund (WCRF) (ref. 12-1280). CZ was supported by a Wellcome Trust studentship (ref. 094885/Z/10/Z). PW is a Cancer Research UK Life Fellow. The funders had no role in the design of the study, the collection, analysis and interpretation of the data, the writing of the paper or the decision to submit the paper for publication.

Author contributions SM, PW, PC and SRS supervised the project. $\mathrm{CZ}$ and SM wrote the paper; CZ, SRS, SM, FR and SE designed the experiments; BA-L supervised and $\mathrm{CZ}$ performed the bioinformatics analysis; RK and JF supported the high- content data acquisition and data extraction; SE designed the xenograft studies, performed by $\mathrm{ADHB} ; \mathrm{CZ}$ performed the majority of experiments; $\mathrm{AH}$ performed assessment and FR analysed the outcome of the pharmacokinetics analysis; ME performed the Ki-67-based response analysis; CZ, SRS, SE, FR and SM analysed data.

\section{Compliance with ethical standards}

Conflict of interest CZ, BA-L, SE, ADHB, FR, AH, PAC and PW are current employees of The Institute of Cancer Research, which has a Rewards to Inventors scheme and has a commercial interest in the development of inhibitors of CDKs, with intellectual property licensed to, and research funding provided by Merck and Cyclacel Pharmaceuticals. PW is a consultant for Astex Pharmaceuticals, CV6 Therapeutics, Nextechinvest and Storm Therapeutics and holds equity in Nextechinvest, Storm Therapeutics and Chroma Therapeutics. BAL consults for or is a member of the advisory board for GSK and Astex
Pharmaceuticals, and she is a former employee of Inpharmatica Ltd. All other authors declare that there is no conflict of interest regarding the content and publication of this article.

Publisher's note Springer Nature remains neutral with regard to jurisdictional claims in published maps and institutional affiliations.

Open Access This article is licensed under a Creative Commons Attribution 4.0 International License, which permits use, sharing, adaptation, distribution and reproduction in any medium or format, as long as you give appropriate credit to the original author(s) and the source, provide a link to the Creative Commons license, and indicate if changes were made. The images or other third party material in this article are included in the article's Creative Commons license, unless indicated otherwise in a credit line to the material. If material is not included in the article's Creative Commons license and your intended use is not permitted by statutory regulation or exceeds the permitted use, you will need to obtain permission directly from the copyright holder. To view a copy of this license, visit http://creativecommons. org/licenses/by/4.0/.

\section{References}

1. Choi YJ, Anders L. Signaling through cyclin D-dependent kinases. Oncogene. 2014;33:1890-903.

2. Sherr CJ, Beach D, Shapiro GI. Targeting CDK4 and CDK6: from discovery to therapy. Cancer Disco. 2016;6:353-67.

3. Parsons DW, Jones S, Zhang X, Lin JC, Leary RJ, Angenendt P, et al. An integrated genomic analysis of human glioblastoma multiforme. Science. 2008;321:1807-12.

4. Cancer Genome Atlas N. Comprehensive molecular portraits of human breast tumours. Nature. 2012;490:61-70.

5. Whittaker SR, Mallinger A, Workman P, Clarke PA. Inhibitors of cyclin-dependent kinases as cancer therapeutics. Pharm Ther. 2017;173:83-105.

6. Hamilton E, Infante JR. Targeting CDK4/6 in patients with cancer. Cancer Treat Rev. 2016;45:129-38.

7. Knudsen ES, Witkiewicz AK. The strange case of CDK4/6 inhibitors: mechanisms, resistance, and combination strategies. Trends Cancer. 2017;3:39-55.

8. O'Leary B, Finn RS, Turner NC. Treating cancer with selective CDK4/6 inhibitors. Nat Rev Clin Oncol. 2016;13:417-30.

9. Rocca A, Schirone A, Maltoni R, Bravaccini S, Cecconetto L, Farolfi A, et al. Progress with palbociclib in breast cancer: latest evidence and clinical considerations. Ther Adv Med Oncol. 2017;9:83-105.

10. Bilgin B, Sendur MAN, Sener Dede D, Akinci MB, Yalcin B. A current and comprehensive review of cyclin-dependent kinase inhibitors for the treatment of metastatic breast cancer. Curr Med Res Opin. 2017;33:1559-69.

11. Schettini F, De Santo I, Rea CG, De Placido P, Formisano L, Giuliano M, et al. CDK 4/6 inhibitors as single agent in advanced solid tumors. Front Oncol. 2018;8:608.

12. Sherr CJ. The Pezcoller lecture: cancer cell cycles revisited. Cancer Res. 2000;60:3689-95.

13. Asghar U, Witkiewicz AK, Turner NC, Knudsen ES. The history and future of targeting cyclin-dependent kinases in cancer therapy. Nat Rev Drug Disco. 2015;14:130-46.

14. Dyson N. The regulation of E2F by pRB-family proteins. Genes Dev. 1998;12:2245-62.

15. Binne UK, Classon MK, Dick FA, Wei W, Rape M, Kaelin WG Jr., et al. Retinoblastoma protein and anaphase-promoting complex physically interact and functionally cooperate during cellcycle exit. Nat Cell Biol. 2007;9:225-32. 
16. Verschuren EW, Jackson PK. Putting transcription repression and protein destruction in pRb's pocket. Dev Cell. 2007;12:169-70.

17. Malumbres M, Sotillo R, Santamaria D, Galan J, Cerezo A, Ortega S, et al. Mammalian cells cycle without the D-type cyclindependent kinases Cdk4 and Cdk6. Cell. 2004;118:493-504.

18. Kozar K, Ciemerych MA, Rebel VI, Shigematsu H, Zagozdzon A, Sicinska E, et al. Mouse development and cell proliferation in the absence of D-cyclins. Cell. 2004;118:477-91.

19. Geng Y, Whoriskey W, Park MY, Bronson RT, Medema RH, Li $\mathrm{T}$, et al. Rescue of cyclin D1 deficiency by knockin cyclin E. Cell. 1999;97:767-77.

20. Satyanarayana A, Kaldis P. Mammalian cell-cycle regulation: several Cdks, numerous cyclins and diverse compensatory mechanisms. Oncogene. 2009;28:2925-39.

21. Franco J, Witkiewicz AK, Knudsen ES. CDK4/6 inhibitors have potent activity in combination with pathway selective therapeutic agents in models of pancreatic cancer. Oncotarget. 2014;5:6512-25.

22. Dean JL, Thangavel C, McClendon AK, Reed CA, Knudsen ES. Therapeutic CDK4/6 inhibition in breast cancer: key mechanisms of response and failure. Oncogene. 2010;29:4018-32.

23. Wang L, Wang J, Blaser BW, Duchemin AM, Kusewitt DF, Liu T, et al. Pharmacologic inhibition of CDK4/6: mechanistic evidence for selective activity or acquired resistance in acute myeloid leukemia. Blood. 2007;110:2075-83.

24. Herrera-Abreu MT, Palafox M, Asghar U, Rivas MA, Cutts RJ, Garcia-Murillas I, et al. Early adaptation and acquired resistance to CDK4/6 inhibition in estrogen receptor-positive breast cancer. Cancer Res. 2016;76:2301-13.

25. Gu J, Xia X, Yan P, Liu H, Podust VN, Reynolds AB, et al. Cell cycle-dependent regulation of a human DNA helicase that localizes in DNA damage foci. Mol Biol Cell. 2004;15:3320-32.

26. Richardson E, Stockwell SR, Li H, Aherne W, Cuomo ME, Mittnacht S. Mechanism-based screen establishes signalling framework for DNA damage-associated G1 checkpoint response. PLoS ONE. 2012;7:e31627.

27. Kitagawa M, Higashi H, Jung HK, Suzuki-Takahashi I, Ikeda M, Tamai K, et al. The consensus motif for phosphorylation by cyclin D1-Cdk4 is different from that for phosphorylation by cyclin $\mathrm{A} / \mathrm{E}-$ Cdk2. EMBO J. 1996;15:7060-9.

28. Ye F, Bauer JA, Pietenpol JA, Shyr Y. Analysis of highthroughput RNAi screening data in identifying genes mediating sensitivity to chemotherapeutic drugs: statistical approaches and perspectives. BMC Genom. 2012;13:S3.

29. Swanton C, Marani M, Pardo O, Warne PH, Kelly G, Sahai E, et al. Regulators of mitotic arrest and ceramide metabolism are determinants of sensitivity to paclitaxel and other chemotherapeutic drugs. Cancer Cell. 2007;11:498-512.

30. Chou TC, Talalay P. Quantitative analysis of dose-effect relationships: the combined effects of multiple drugs or enzyme inhibitors. Adv Enzym Regul. 1984;22:27-55.

31. Leontieva OV, Blagosklonny MV. CDK4/6-inhibiting drug substitutes for $\mathrm{p} 21$ and p16 in senescence: duration of cell cycle arrest and MTOR activity determine geroconversion. Cell Cycle. 2013;12:3063-9.

32. Blagg J, Workman P. Choose and use your chemical probe wisely to explore cancer biology. Cancer Cell. 2017;32:9-25.

33. Bunz F, Dutriaux A, Lengauer C, Waldman T, Zhou S, Brown JP, et al. Requirement for p53 and p21 to sustain G2 arrest after DNA damage. Science. 1998;282:1497-501.
34. Scholzen T, Gerdes J. The Ki-67 protein: from the known and the unknown. J Cell Physiol. 2000;182:311-22.

35. Finn RS, Dering J, Conklin D, Kalous O, Cohen DJ, Desai AJ, et al. PD 0332991, a selective cyclin D kinase 4/6 inhibitor, preferentially inhibits proliferation of luminal estrogen receptorpositive human breast cancer cell lines in vitro. Breast Cancer Res. 2009;11:R77.

36. Gu Y, Rosenblatt J, Morgan DO. Cell cycle regulation of CDK2 activity by phosphorylation of Thr160 and Tyr15. EMBO J. 1992;11:3995-4005.

37. Frescas D, Pagano M. Deregulated proteolysis by the F-box proteins SKP2 and beta-TrCP: tipping the scales of cancer. Nat Rev Cancer. 2008;8:438-49.

38. Gentile A, Trusolino L, Comoglio PM. The Met tyrosine kinase receptor in development and cancer. Cancer Metastas- Rev. 2008;27:85-94.

39. Engels B, Rowley DA, Schreiber H. Targeting stroma to treat cancers. Semin Cancer Biol. 2012;22:41-49.

40. Matsumoto K, Nakamura T. Hepatocyte growth factor and the Met system as a mediator of tumor-stromal interactions. Int $\mathbf{J}$ Cancer. 2006;119:477-83.

41. Goel S, Wang Q, Watt AC, Tolaney SM, Dillon DA, Li W, et al. Overcoming therapeutic resistance in HER2-positive breast cancers with CDK4/6 inhibitors. Cancer Cell. 2016;29:255-69.

42. Cabodi S, Di Stefano P, Leal Mdel P, Tinnirello A, Bisaro B, Morello V, et al. Integrins and signal transduction. Adv Exp Med Biol. 2010;674:43-54.

43. Bryant $\mathrm{P}$, Zheng Q, Pumiglia K. Focal adhesion kinase controls cellular levels of p27/Kip1 and p21/Cip1 through Skp2-dependent and -independent mechanisms. Mol Cell Biol. 2006;26:4201-13.

44. Bond M, Sala-Newby GB, Newby AC. Focal adhesion kinase (FAK)-dependent regulation of S-phase kinase-associated protein2 (Skp-2) stability. A novel mechanism regulating smooth muscle cell proliferation. J Biol Chem. 2004;279:37304-10.

45. Zhao JH, Reiske H, Guan JL. Regulation of the cell cycle by focal adhesion kinase. J Cell Biol. 1998;143:1997-2008.

46. Huang G, Ho B, Conroy J, Liu S, Qiang H, Golubovskaya V. The microarray gene profiling analysis of glioblastoma cancer cells reveals genes affected by FAK inhibitor Y15 and combination of Y15 and temozolomide. Anticancer Agents Med Chem. 2014;14:9-17.

47. Golubovskaya VM. Targeting FAK in human cancer: from finding to first clinical trials. Front Biosci (Landmark Ed). 2014;19:687-706.

48. Roy-Luzarraga M, Hodivala-Dilke K. Molecular pathways: endothelial cell FAK-A target for cancer treatment. Clin Cancer Res. 2016;22:3718-24.

49. Lv PC, Jiang AQ, Zhang WM, Zhu HL. FAK inhibitors in cancer, a patent review. Expert Opin Ther Pat. 2018;28:139-45.

50. Shibue T, Brooks MW, Weinberg RA. An integrin-linked machinery of cytoskeletal regulation that enables experimental tumor initiation and metastatic colonization. Cancer Cell. 2013;24:481-98.

51. Carpenter AE, Jones TR, Lamprecht MR, Clarke C, Kang IH, Friman O, et al. CellProfiler: image analysis software for identifying and quantifying cell phenotypes. Genome Biol. 2006;7: R100.

52. Workman P, Balmain A, Hickman JA, McNally NJ, Rohas AM, Mitchison NA, et al. UKCCCR guidelines for the welfare of animals in experimental neoplasia. Lab Anim. 1988;22:195-201. 